\title{
Conservation Agriculture-Based Maize-Bean Cropping Systems in South Central Ethiopia
}

\author{
Goshime Muluneh Mekasha $^{1 *}$ Solomon Admassu Seyoum ${ }^{1} \quad$ Walter Tamuka Mupangwa ${ }^{2}$ \\ Alemayehu Zemede Lemma ${ }^{1} \quad$ Haimanot Beruk Senbeta $^{1} \quad$ Mahmud Aman Tello $^{1}$ \\ Mesele Haile onu ${ }^{1}$ Tesfaye Hailu Estifanose ${ }^{3}$ \\ 1.Ethiopian Institute of Agricultural Research, Hawassa Maize Research Sub-Centre, PO Box 845, Hawassa, \\ Ethiopia \\ 2.International Maize and Wheat Improvement Center (CIMMYT), Shola Campus, ILRI, PO Box 5689, Addis \\ Ababa, Ethiopia \\ 3.Wolaita Sodo University, Hydraulics and Hydro Power Engineering, PO Box 138, Sodo, Ethiopia
}

\begin{abstract}
Conservation agriculture (CA) is defined as sustainable agriculture production system comprising a set of farming practices adapted to the requirement of crops and local condition of each region. This experiment was conducted at three districts: East-Badawaho, Meskan and Hawassa-Zuriya. In each district five farmers hosted the experiment and each farmer was used as a replicate. The experiment was run for six years (2011-2016) at each farmer's field. The experiment consisted of five treatments (Continuous sole maize, Maize bean rotation, Maize-bean intercropping, Bean rotation under CA and farmer practice. BH-543 maize variety and Hawassa-Dume common bean variety were used. Soil moisture content under CA practices was higher than the farmer practice during drought year. Maize yield and yield related traits and soil water data were collected from each site. At East-Badawacho grain yield was $4 \%$ higher in CA compared with farmer practice. Maize bean rotation and sole maize under CA out yielded the farmer practice by 13 and 4\%, respectively but maize-bean inter-cropping had 5\% lower grain yield. At Hawassa-Zuriya, CA maize bean rotation had higher yield than farmer practice in 2011 and 2013. At Meskan, CA had 8\% higher than FP. Maize-bean inter-cropping, maize bean rotation and sole maize under CA had 10,8 and $6 \%$ higher grain yield than farmer practice, respectively. Common bean grain yield from bean rotation under CA had 2799, 2908, and $3226 \mathrm{~kg} \mathrm{ha}^{-1}$ at East-Badawacho, Hawassa-Zuriya and Meskan district respectively. Intercropping under CA had 817, 1065 and $927 \mathrm{~kg} \mathrm{ha}^{-1}$, respectively. Generally, CA cropping systems had random drought stress reduction potential as compared with common practice and even under normal condition cropping systems under CA had higher grain yield and biomass production potential.
\end{abstract}

Keywords: cropping systems, farmer practice, sole maize, rotation, inter cropping; rift valley

DOI: $10.7176 / \mathrm{JNSR} / 10-12-01$

Publication date:June 30th 2020

\section{INTRODUCTION}

In Africa, agriculture sector is mainly in the hands of small-scale farmers who use traditional methods and tools of production (Musa 2015). Agricultural production in the semi-arid regions of Sub-Saharan Africa (SSA) is challenged by many risk factors and high vulnerability of poorly resourced farmers (Solomon 2018). Key sources of risk in agriculture include climate, socio-economic factors, soil degradation, and poorly developed markets (Kassie et al. 2013). Agriculture continues to be the major sector in Ethiopia's economy, with cereals playing a critical role and maize is Ethiopia's largest cereal commodity in terms of total production, acreage, and the number of farm holdings (Rashid et al. 2010). Rainfall in Ethiopia is seasonal with high spatial and temporal variability. In the Central and Southern Rift Valley of Ethiopia rainfall pattern is bimodal and starts with the spring rains or Belg during the months of March-May and the summer rain or Kiremt extend from June-September (Solomon 2018).

Conservation agriculture (CA) is characterized by minimum soil disturbance, diversified crop rotations, and surface crop residue of soil and environmental degradation while sustaining crop production (Farooq and Siddique, 2015).Soil Erosion involves both the losses of the soil itself and loss of organic and material nutrients found in the soil (Trippathi and Singh 1993) and in organic matter (Geremaw 2005). Soil can be eroded away by wind and water (Semu 2018). Soil erosion affects an estimated 1,100 million hectares of land worldwide resulted the transport of 2.0 -2.5 Mg of soil to the oceans each year (MOA 2015). Erosion removes the most productive portion of the soil, that is, the chemically active part such as organic matter and clay fraction (Semu 2018). It also causes a deterioration of soil structure, moisture holding capacity through lowering soil depth, incising bulk density, soil crusting, and reducing water infiltration. Similarly soil erosion is one of the principal environmental problems in Ethiopia and Eritrea resulting in decreasing productivity of farmlands (Hurni 1987) and 2 million hectares of land in Ethiopia has been severely degraded (Shiferaw 2005).

The prevention of erosion on cultivated land and other areas depends essentially on the reduction of soil detachment and runoff on the maintenance of adequate vegetation ground cover (Fitsum 2002). Soil conservation 
involves the various methods used to reduce soil erosion to prevent depletion of soil nutrients and soil moisture and to enrich the nutrients status of the soil. The conservation techniques include terracing and others. Leaving crop residues in the field will reduce the wind speed from the farm yard also uses to reduce evapotranspiration so that conserve moisture because it acts as a wind break and soil cover of the soil surface (Semu 2018). For sustainable agriculture one of the major steps is effective soil and water conservation (Fitsum 2002). Soil conservation needs to be considered in line with the soil erosion control measures (Geremaw 2005). Thus, for effective soil conservation a good soil cover needs a protected soil from erosion factors, a good soil cover which in turn increases the fertility of the soil leading to sustainable agricultural development. Sustainable use and management of water leads to developed agriculture which in turn leads to sustainable agricultural production and development (Herweg 1999). Therefore, soil and water conservation play a major role in sustainable agricultural production as the conservation of the two is necessary for wise resource development especially in agricultural sector.

Cultivation and tillage play an important role in agriculture. The benefits of tillage in agriculture are explored before introducing conservation tillage (CT), a practice that was borne out of the American dust bowl of the 1930s. $\mathrm{CA}$ with no till, mulch and rotations significantly improve soil properties and other biotic factors. CA is a more sustainable and environmentally friendly management system for cultivating crops (Hobbs et al. 2008) and Hobbs and his co-authors suggested that agriculture should sustainably produce more food from less land through more efficient use of natural resources and with minimal impact on the environment in order to meet growing population demands. Promoting and adopting CA management systems can help meet this goal. The scientific community pointed out as the member of farming community have been advocating a move to reduced tillage systems that use less fossil fuel, reduce run-off and erosion of soils and reverse the loss of soil organic matter since the 1930s, during the following 75 years.

Conservation agriculture represents asset of three crop management principles:(1) direct planting of crops with minimum soil disturbance (no-till), (2) permanent soil cover by crop residues or cover crops, and (3) crop rotation (Hobbs et al. 2008; FAO 2011). Conservation agriculture (CA) aims to conserve, improve and make more efficient use of natural resources through integrated management of available soil, water and biological resources combined with external inputs. It contributes to environmental conservation as well as to enhanced and sustained agricultural production. In recent decades, widespread adoption of no-till has occurred over approximately 125 million hectares, equivalent to $9 \%$ of global arable land, with varying degrees of application of the other two conservation agriculture principles (FAO 2011; Friedrich et al. 2012). Millions of hectares in dry climates of subSaharan Africa and South Asia have recently been identified as suitable for sustainable intensification efforts (ICARDA 2014). To make conservation agriculture is to be successful at increasing crop productivity in these areas; it must be adjusted to local conditions through an innovative, multi-stakeholder driven approach that is sensitive to market opportunities, equipment availability, and farmers' production objectives and needs (Erenstein et al. 2012).

The retention of $30 \%$ surface cover by residues characterizes the lower limit of classification for conservation tillage, but other conservation objectives for the practice include conservation of time, fuel, earthworms, soil water, soil structure and nutrients (Hobbs et al. 2008). Conservation tillage is a set of practices that leave crop residues on the surface which increases water infiltration and reduces erosion (Hobbs et al. 2008). Thus, residue levels alone do not adequately describe all conservation tillage practices.

Conservation agriculture (CA) aims to conserve, improve and make more efficient use of natural resources through integrated management of available soil, water and biological resources combined with external inputs. It contributes to environmental conservation as well as to enhanced and sustained agricultural production. It can also be referred to as resource efficient or resource effective agriculture (FAO). No-tillage minimizes soil organic matter losses and is a promising strategy yield to maintain or even increase soil C and N stocks (Bayer et al. 2000). The benefits of CA especially when rotated with leguminous crops, increase over time, suggesting that there are improvements in soil structure and fertility (Thierfelder et al. 2012)

The role of surface residues on water conservation and indicates that this association between surface residues, enhanced water infiltration and evaporation led to the adoption of CA after the 1930s (Unger et al. 1988). Surface mulch helps reduce water losses from the soil by evaporation and also helps moderate soil temperature and it used promotes biological activity and enhances nitrogen mineralization, especially in the surface layers (Hatfield \& Pruegar 1996; Hobbs et al. 2008;). Infiltration of water under long-term (8-10 years) conservation tillage (zero and subsurface tillage with residue retention) was higher compared to conventional tillage (frequent plowing plus no residue retention) on a grey cracking clay and a sandy loam soil in south-eastern Australia (Bissett \& O'leary 1996).

Crop rotation is an agricultural management tool with ancient origins (Hobbs et al. 2008). Rotation is cultural control of plant diseases from an historical view (Howard 1996). The rotation of different crops with different rooting patterns combined with minimal soil disturbance in zero-till systems promotes a more extensive network of root channels and macrospores in the soil and this helps in water infiltration to deeper depths (Hobbs et al. 2008). 
Because rotations increase microbial diversity, the risk of pests and disease outbreaks from pathogenic organisms is reduced, since the biological diversity helps keep pathogenic organisms in check (Leake 2003).

Producers and investigators conducted different cropping systems to increase productivity and sustainability by practicing crop rotations, relay cropping, and inter-cropping of annual cereals with legumes (Yilmaz et al. 2008). Inter-cropping is a type of mixed cropping and defined as the agricultural practice of cultivating two or more crops in the same space at the same time. It increases in productivity per unit of land via better utilization of resources, minimizes the risks, and stabilizes the yield (Ananthi et al. 2017). Inter-cropping of cereals with legumes has been popular in tropics ( Tsubo et al., 2005) and rain-fed areas of the world (Agegnehu et al. 2006; Dhima et al. 2007) due to its advantages for soil conservation (Ananthi et al. 2017) and lodging resistance (Anil et al. 1998), weed control (Banik et al. 2006; Ananthi et al. 2017 ), yield increment (Chen et al. 2004), hay curing, forage preservation over pure legumes, high crude protein percentage and protein yield and legume root parasite infections control (Fenandez-Aparicio et al. 2007).

Legumes, such as common vetch, common bean and cowpea are extensively used in inter-cropping with cereals in Turkey (Akman and Sencar 1999; Yilmaz et al. 2008), Finger millet with maize (Nath 2016), wheat with Soybean (Sandler and Kelly 2016).

The objective of the study was to answer the questions like Does cropping systems under CA have potential crop production benefit and soil moisture holding capacity as compared with conventional practice? This study specific objective was targeted to evaluate and compare maize bean cropping systems under conventional and conservation tillage practices in addition to assessing soil moisture holding capacity of different cropping systems. The other objective of the study was to know the advantage of cropping systems under CA in reduction of risks from crop failure as compared with conventional practice due to moisture stress during the cropping season.

\section{Materials and Method}

\subsection{Description of the Study Area}

The experiment was conducted at East-Badawacho (alt 1788 masl), Meskan (alt 1839 masl) and Hawassa-Zuriya (alt 1696 masl) districts farmers' fields during the period between 2011 and 2016 cropping seasons under rain-fed in the southern Ethiopia. The common soil types at east Badawacho, Meskan and Hawassa-Zuriya are black basaltic soils (Vertisols), eutric Cambisols and vitric Andosols, respectively (Addise, 2014; Getahun et al, 2014; Lemma et al., 2015). These areas are characterized by bimodal rainfall received between March and September. These areas are characterized by bimodal rainfall received between March and September. The cumulative annual rainfall ranges between 872 to $1322 \mathrm{~mm}$ at East-Badawacho, 815 and $1346 \mathrm{~mm}$ at Meskan, and 900 and $1400 \mathrm{~mm}$ at Hawassa-Zuriya (source: TAMSAT). These areas are characterized by erratic rainfall distribution. The daily and cumulative monthly rainfall for sites is given in the Figs. 2-4.

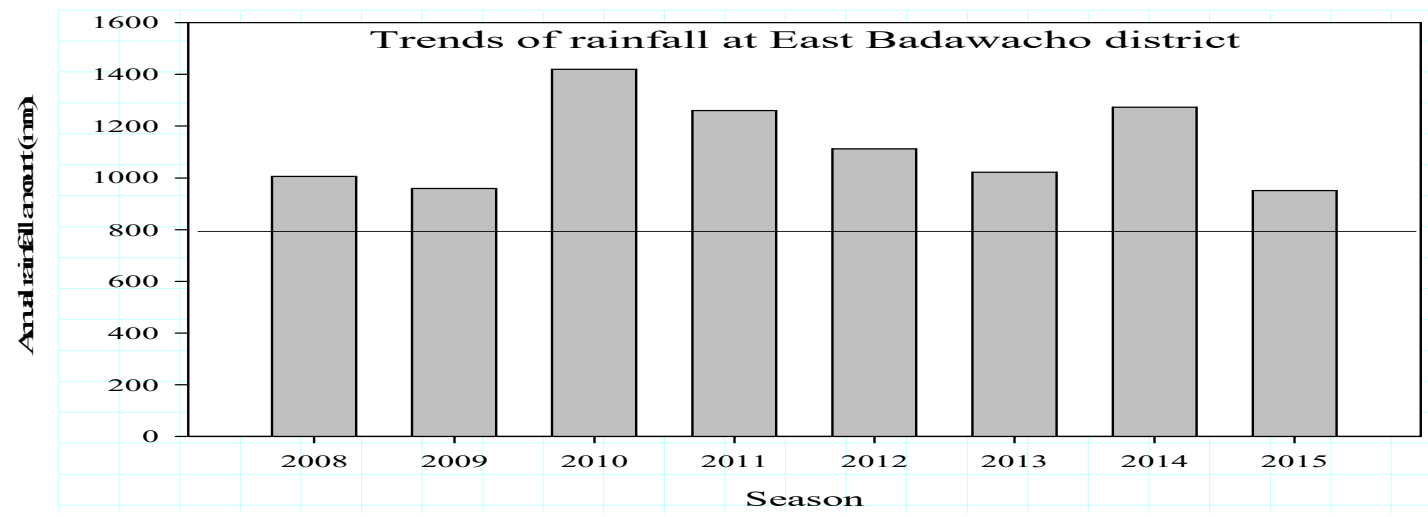

Fig.1. 


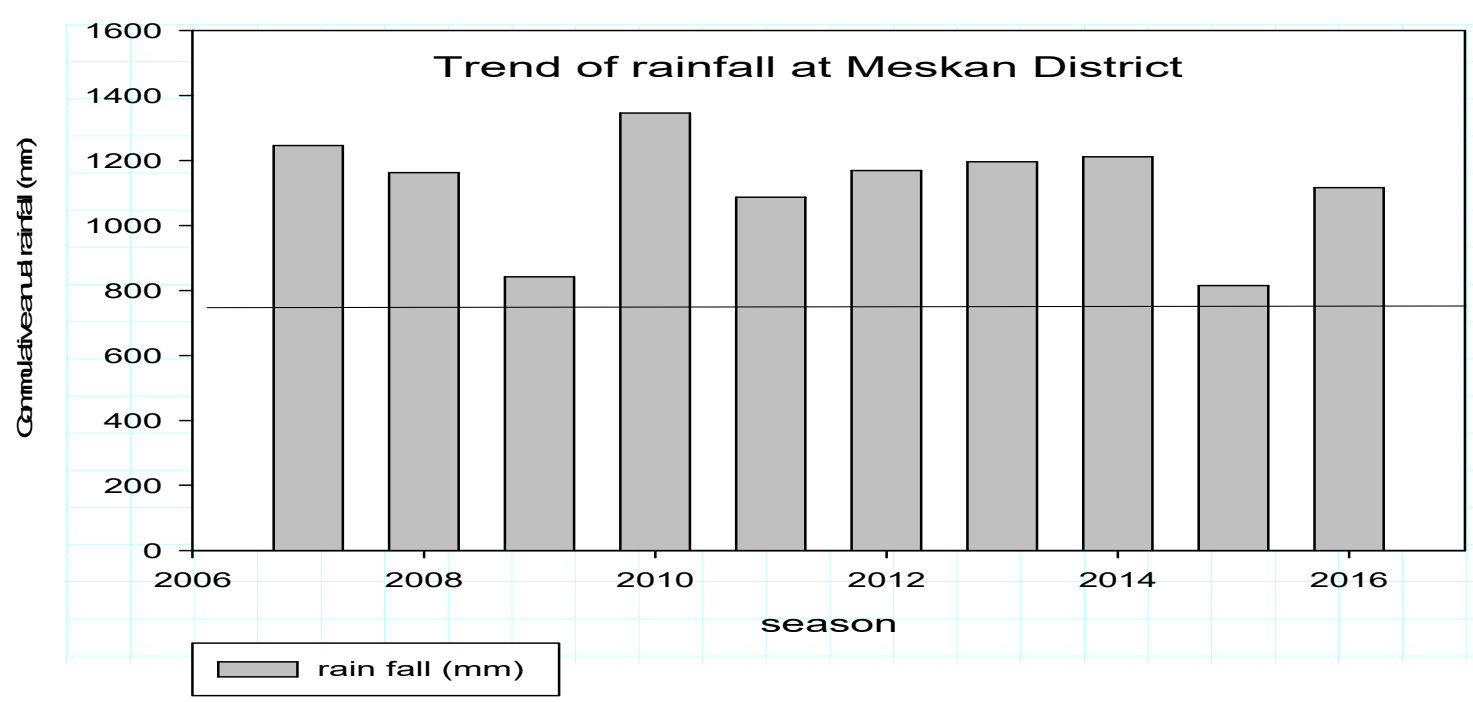

Fig. 2.

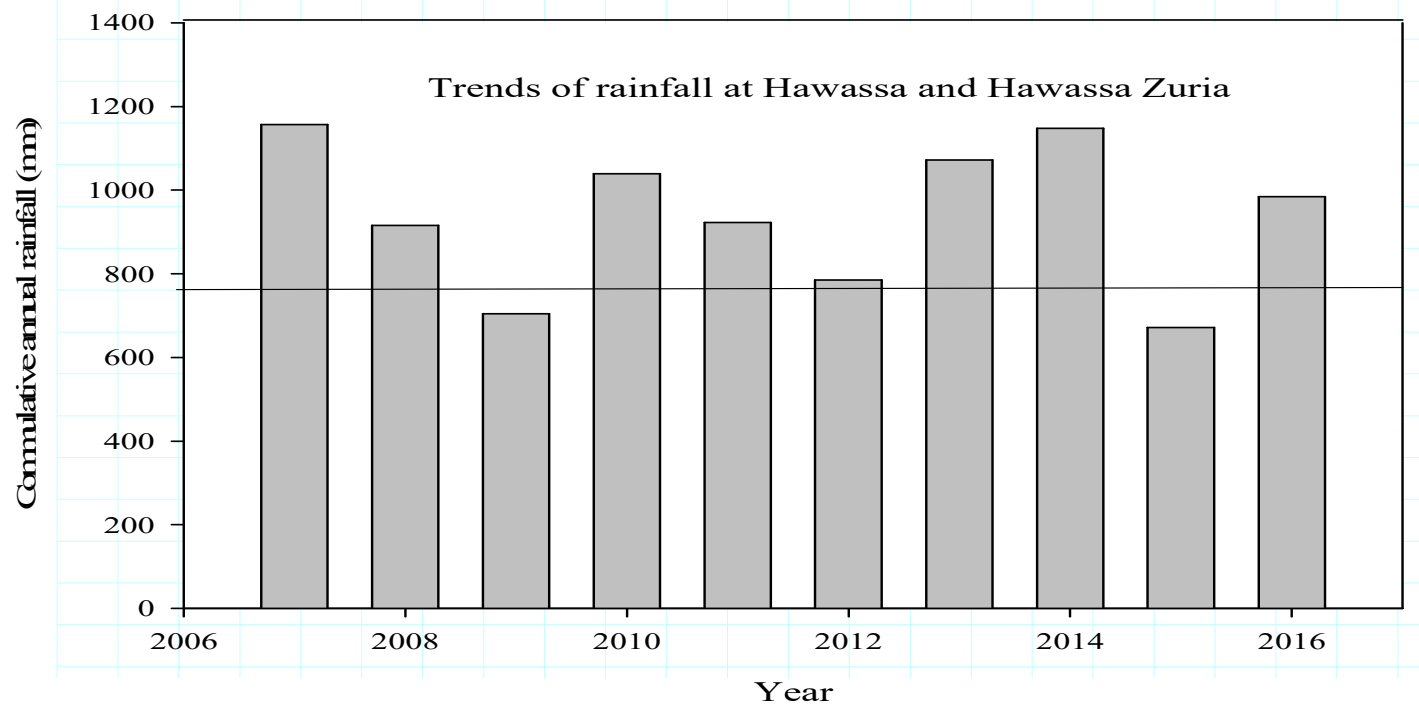

Fig.3. 


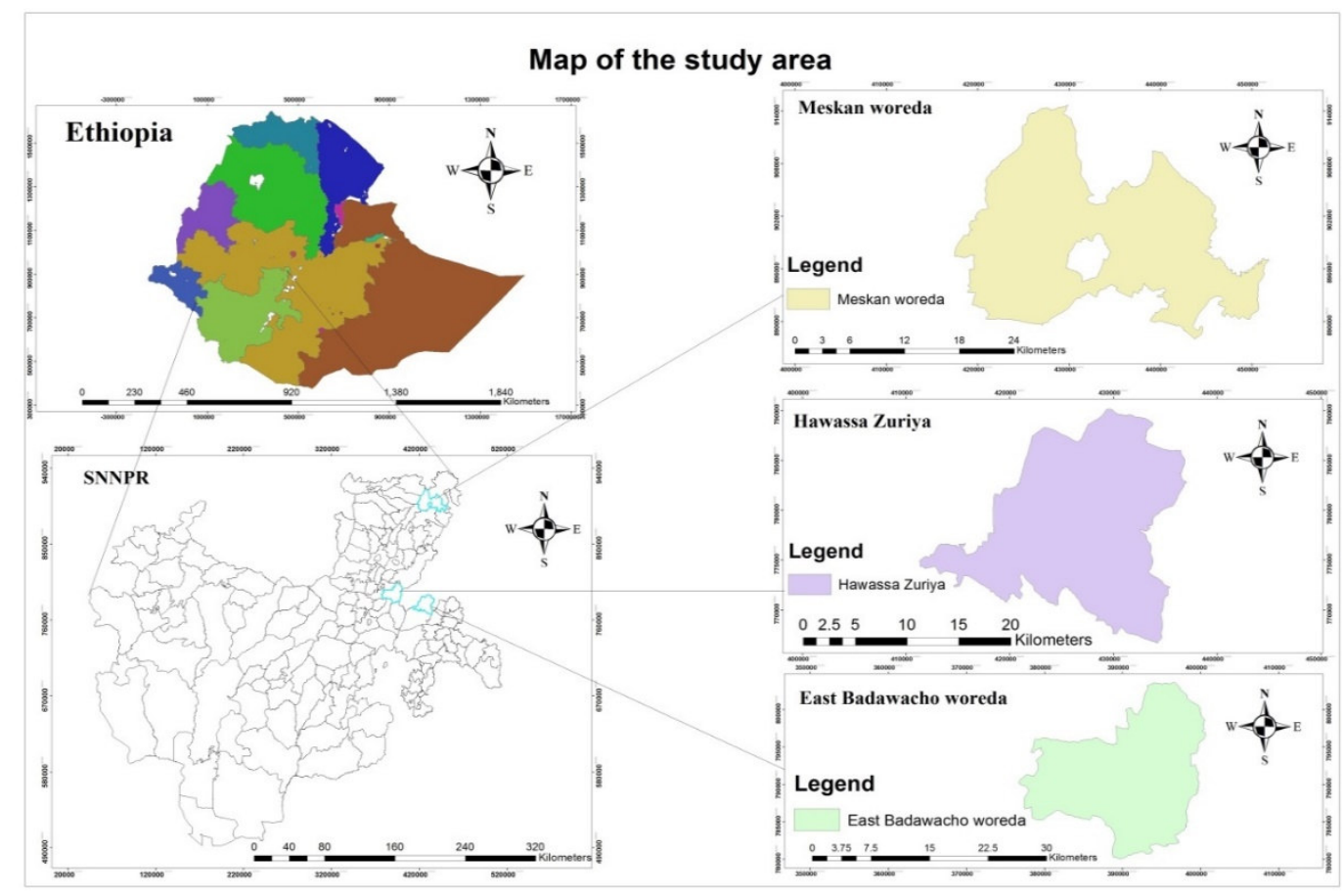

Fig.4

Rainfall data at East Badawacho district

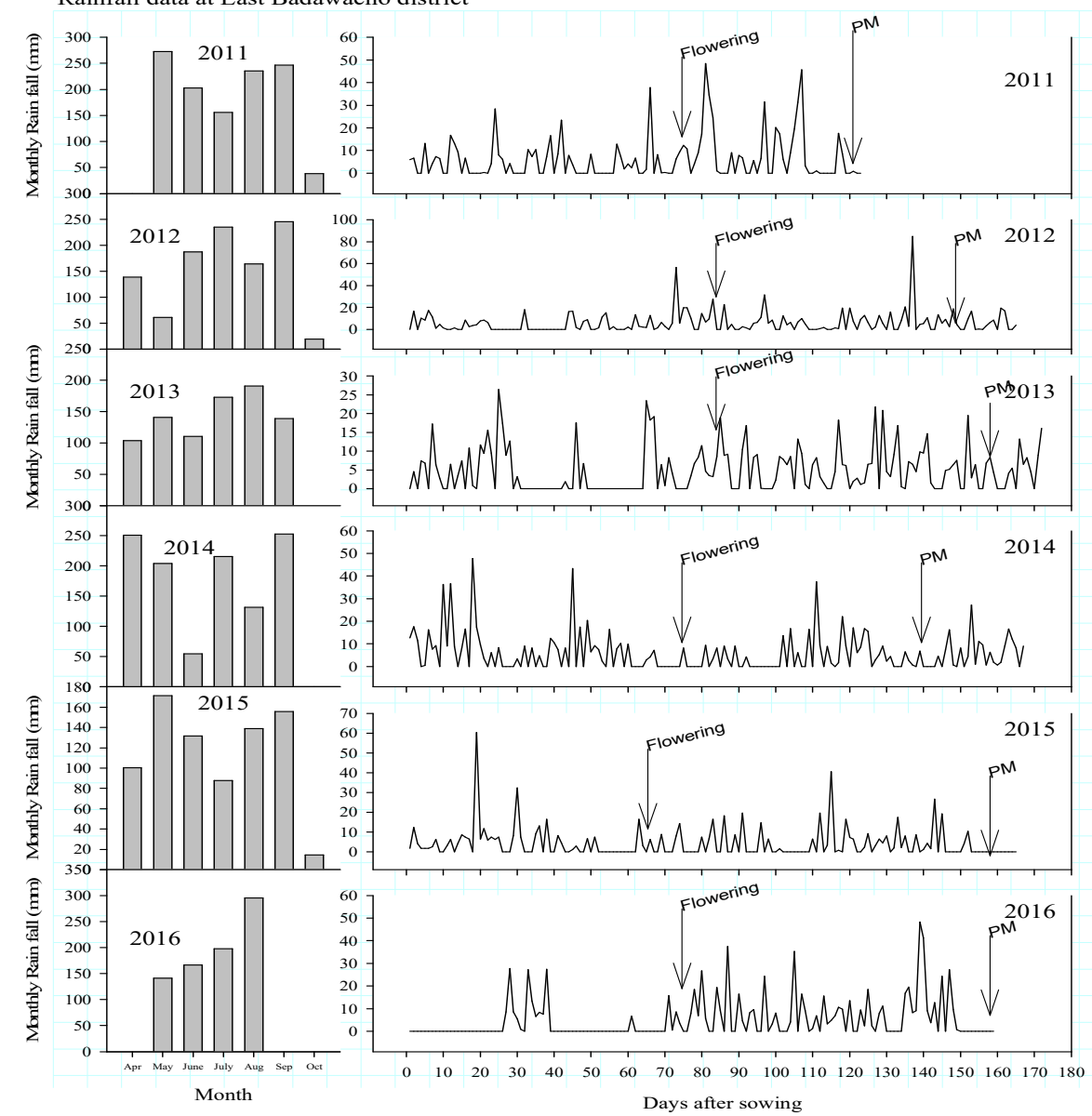

Fig.5.Cumulative monthly rainfall (bar grapha) and daily rainfall distribution (line graph) during 2011 - 2016 cropping seasons at East-Badawacho. The arrows indicate flowering and physiological maturity (PM) stages of the crop. The insert line graph indicates the cumulative rainfall 15 days before flowering (15 dbf), 15 days after flowering (15 daf), before flowering (Bf) and, after flowering (Af) during the cropping season 


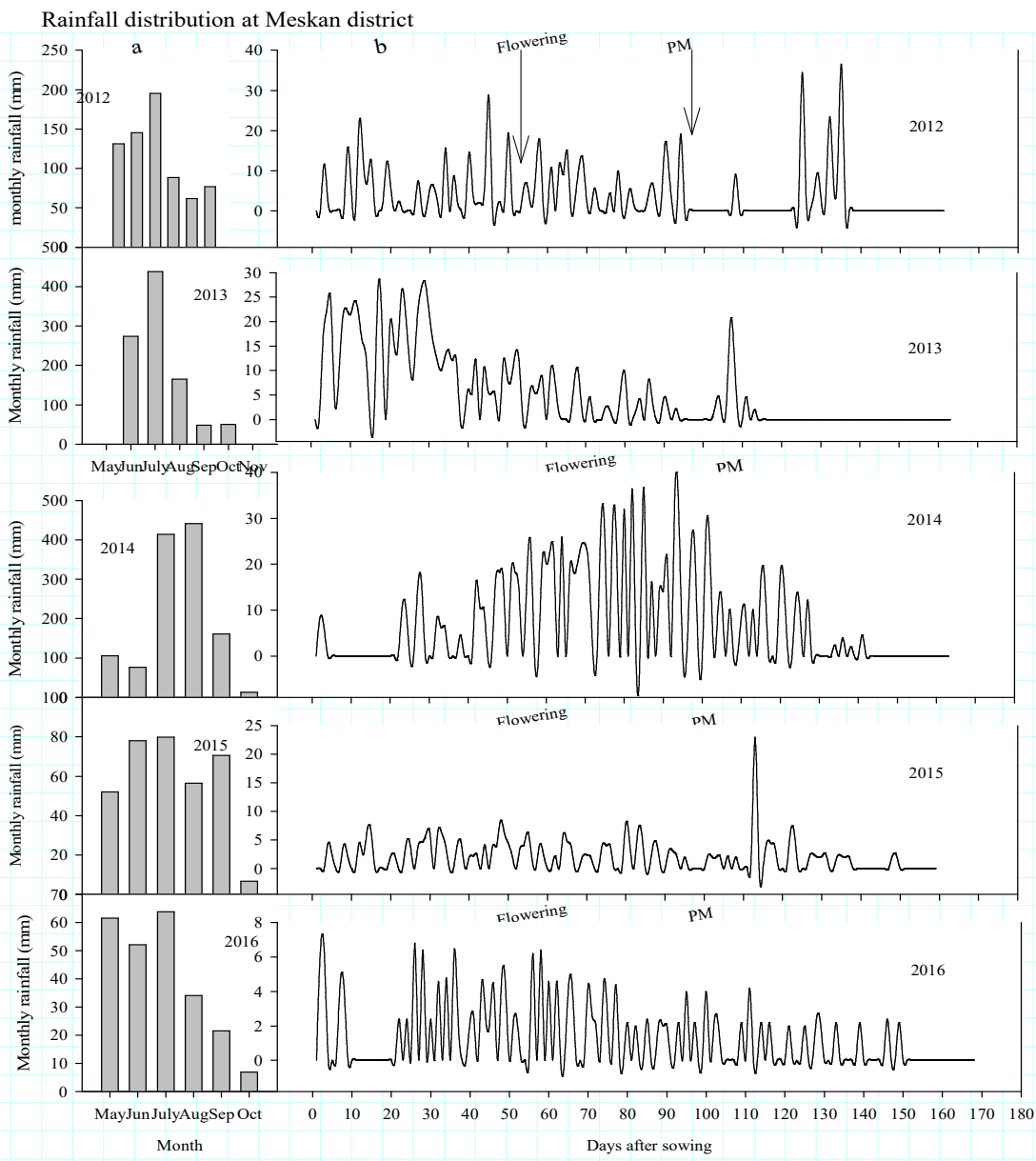

Fig.6. Cumulative monthly rainfall (bar graph) and daily rainfall distribution (line graph) during 2011 and 2016 cropping seasons at Meskan district. The arrows indicate flowering and physiological maturity (PM) stages of the crop. The inset on line graph indicate the cumulative rainfall 15 days before flowering (15 dbf), 15 days after flowering (15 daf), before flowering (Bf) and after flowering (Af) during the cropping season. 


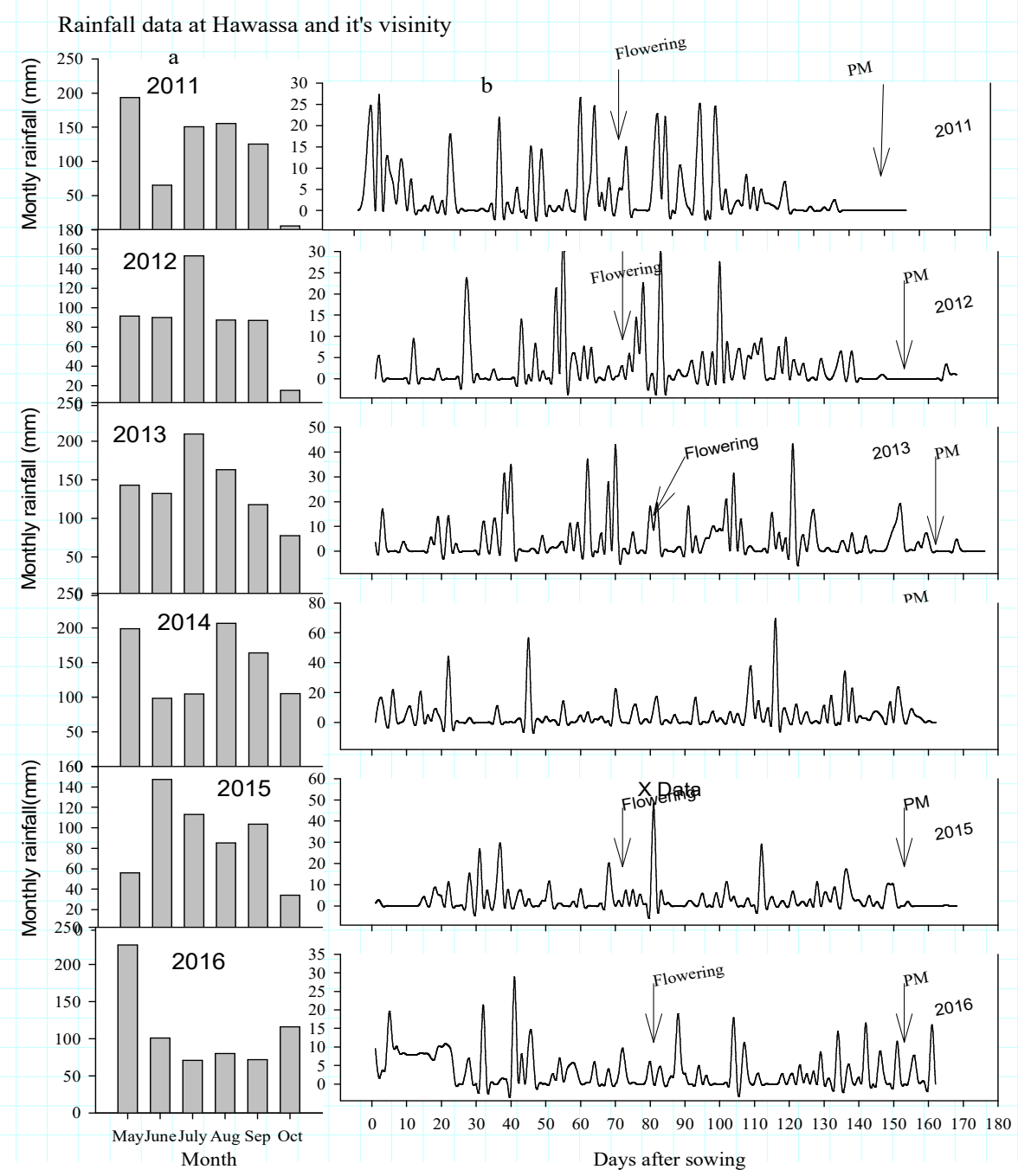

Fig.7. Cumulative monthly rainfall (bar graph) and daily rainfall distribution (line graph) during 2011 to 2016 cropping seasons at Hawassa-Zuriya district. The arrows indicate flowering and physiological maturity (PM) stages of the crop. The inset on line graph indicate the cumulative rainfall 15 days before flowering (15 dbf), 15 days after flowering (15 daf), before flowering (Bf) and after flowering (Af) during the cropping season. Table 1: Rainfall amount $(\mathrm{mm})$ at different cropping time in each year

\begin{tabular}{llcccccc}
\hline & Time & 2011 & 2012 & 2013 & 2014 & 2015 & 2016 \\
\hline East-Badawacho & 15days before flowering & 83.3 & 50.3 & 60.9 & 42.3 & 18.2 & 6.7 \\
& 15days after flowering & 158.4 & 86.3 & 86.8 & 42.5 & 44.9 & 131.5 \\
& before flowering & 329.0 & 257.6 & 275.8 & 513.5 & 291.3 & 148.2 \\
& after flowering & 224.2 & 576.1 & 533.4 & 459.0 & 332.1 & 624.8 \\
& Cropping time & 553.2 & 833.7 & 809.2 & 972.5 & 623.4 & 773.0 \\
\hline \multirow{2}{*}{ Meskan } & 15days before flowering & - & 98.6 & 59.9 & 234.8 & 37.6 & 37.2 \\
& 15days after flowering & - & 67.3 & 25.3 & 211.8 & 41.6 & 16.8 \\
& before flowering & - & 362.3 & 742.2 & 505.6 & 163.8 & 128.4 \\
& after flowering & - & 224.5 & 98.2 & 614.6 & 159.2 & 71.2 \\
& Cropping time & - & 586.8 & 840.4 & 1120.2 & 323.0 & 199.6 \\
\hline \multirow{3}{*}{ Hawassa-Zuriya } & 15days before flowering & 83.7 & 65.6 & 146.5 & 35.3 & 23.3 & 29.9 \\
& 15days after flowering & 77.5 & 69.2 & 56.4 & 57.0 & 53.2 & 45.9 \\
& before flowering & 316.8 & 174.3 & 352.2 & 277.2 & 189.3 & 339.3 \\
& after flowering & 216.7 & 234.6 & 386.8 & 503.5 & 324.1 & 211.3 \\
& Cropping time & 533.5 & 408.9 & 739.0 & 780.7 & 513.4 & 550.6 \\
\hline
\end{tabular}




\subsection{Treatments}

A trial comprising of five treatments was established at each farmer's field in 2011 cropping using farmers as a replicate. The treatments include:

Continuous sole maize,

Bean-Maize rotation,

Maize-bean rotation and

Maize-bean inter-cropping all under conservation agriculture (CA) and

Conventional continuous sole maize (Farmer practice)

For treatments under CA, narrow rows were opened with a hand-hoe to a depth of about $10 \mathrm{~cm}$ to place seeds and basal fertilizer application without prior tillage of the soil and retention of all the maize and bean crop residue produced the previous season as surface mulch). The conventional tillage practice or farmer practice was cultivated similar to the traditional farmers' land preparation practice for maize at each district. Land was prepared by conventional ploughing with an ox-drawn traditional plough called Maresha (ploughed the land 2 - 4 times depending on the soil types) just before planting and long time before planting to loosen the soil.

\subsection{Crop husbandry}

Maize was planted at a spacing of $0.75 \mathrm{~m}$ between rows and $0.30 \mathrm{~m}$ between hills, and common bean was planted at a spacing of $0.40 \mathrm{~m}$ between rows and $0.1 \mathrm{~m}$ between hills. Each plot consisted of 13 rows of $10 \mathrm{~m}$ long (100 $\mathrm{m} 2$ area). Two seeds were planted per hill and later thinned to one seedling upon stand establishment to maintain 44,444 plants ha-1 for maize and 250,000 plants ha-1 for common bean.

All treatments received fertilizer rates recommended: $110 \mathrm{~kg} \mathrm{~N}$ and $46 \mathrm{~kg} \mathrm{P}_{2} \mathrm{O}_{5} \mathrm{ha}^{-1}$ for maize and $46 \mathrm{~kg} \mathrm{P}_{2} \mathrm{O}_{5}$ and $37 \mathrm{~kg}$ of $\mathrm{N} \mathrm{ha}^{-1}$ for common bean. For maize, all the phosphorous and a third of $\mathrm{N}$ was applied as basal dose while two-third $\mathrm{N}$ was side-dressed at 35 days after emergence. For common bean, all the fertilizer was applied at planting. Maize (cv BH-543 (154 days maturity)) and common bean (cv Hawassa Dume (102 days maturity)) varieties, were used in all years. In the maize-bean intercropping treatment, bean was planted at the same time as maize, between maize rows.

The treatments managed through conservation agriculture were sprayed with a broad-spectrum systemic herbicide (glyphosate) 10 days before planting at the rate of 3-liter ha ${ }^{-1}$ to control weed and all plots were maintained weed free afterwards by hand weeding. Whereas, the conventional farmer practice was hand weeded following the common practice done by farmers.

\subsection{Measurements}

\subsubsection{NDVI}

Normalized Difference Vegetative Index (NDVI) was measured at vegetative and flowering stages using a Green Seeker ${ }^{\mathrm{TM}}$ Handheld Optical Sensor Unit (NTech Industries, Inc., USA) following the method used by Govaerts $e t$ al. (2007) and Verhulst et al. (2011) in 2016 for only for treatments at East Badawacho.

\subsubsection{Biomass}

Above-ground biomass was measured at physiological maturity of maize from ten sample plant cut at ground level for fresh biomass measurement. From these ten sample plants $0.5 \mathrm{~kg}$ sub sample was taken before oven drying for dry maize biomass weight measurement. For common bean ten plants were cut at the ground level and dried for biomass. Biomass samples were dried in a fan-circulated oven set at $65^{\circ} \mathrm{C}$ until constant weight and expressed on dry weight basis (Karim et al., 2000).

\subsubsection{Yield and yield components for the component crops}

Grain yield, pods per plant and number of seeds per pod were for common bean. Plants in the middle 11 rows, from an area of $82.5 \mathrm{~m} 2$ were hand harvested at physiological maturity. Ears were shelled, grain weight and grain moisture content measured, and yield was adjusted for $12.5 \%$ grain moisture content. For common bean, total number of pods per plant (PPP) and seeds per pod (SPP) were counted from ten plants and ten pods, respectively. The yield data was then adjusted to $10 \%$ moisture content for common.

2.2.4. Soil sample

Composite soil samples from three cores were taken at three depths, 0-15 cm, 15-30 cm and 30-45 cm, at planting, bean harvesting and maize harvesting every year. The soil samples from each plot were weighed immediate after sampling and oven-dried for 48 hours at $105^{\circ} \mathrm{C}$ for final dry weight.

\subsubsection{Statistical analysis}

Normality of data were checked prior to analysis of variance (ANOVA) using Shapiro-Wilk normality test. Analysis of variance (ANOVA) for each year was done for yield and other traits using SAS version 9.0. Analysis was done for each year independently and for all combined years. Means were separated using LSD test. Graphs were developed using sigma plot 10.0 (Systat Software, San Jose, CA). 


\section{Results and discussion}

\subsection{Soil moisture}

The soil moisture content at planting was significant for treatments only in 2013 at $15-30 \mathrm{~cm}$ depth (Table 2). The highest soil moisture content was observed for maize bean rotation compared with other treatments. At bean harvesting significant difference was observed in 2015 cropping season at $0-15 \mathrm{~cm}$ depth with the highest moisture for bean-maize rotation compared with other treatments (Table 2). Soil moisture at maize harvesting, however, was significant in 2013 and 2015 at $>30 \mathrm{~cm}$ depth, and in 2016 at $0-15 \mathrm{~cm}$ depth.

The soil moisture contents at planting, bean harvesting and maize harvesting were highest in 2016 compared with other seasons (Figs. 8-10). The substantial variation between treatments under CA, rotation and intercropping, and farmers practice was observed at maize harvesting (Fig. 10).

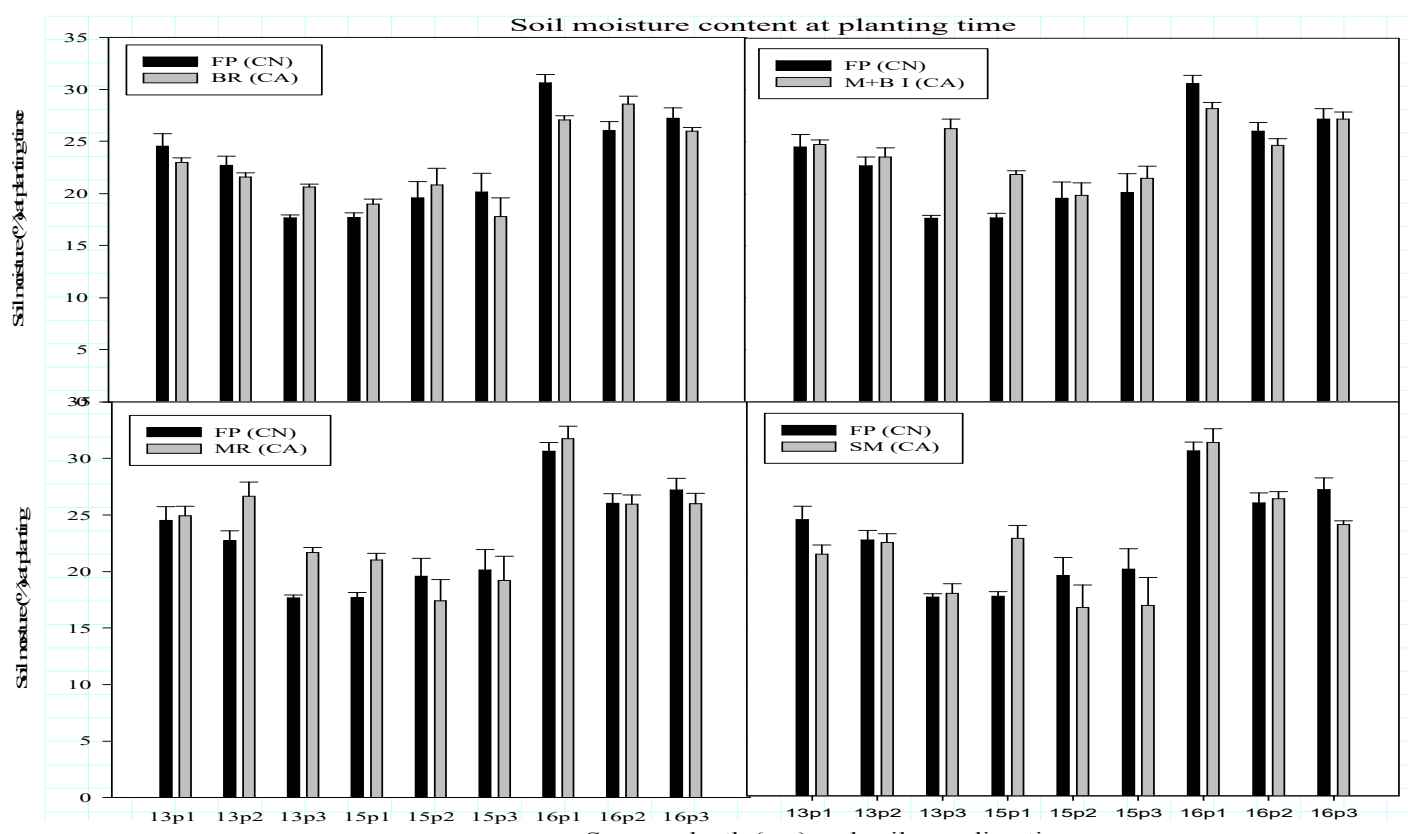

Fig. 8. Soil moisture content in percentage at planting time at three soil depth (depth $1=0-15 \mathrm{~cm}$, depth 2=15$30 \mathrm{~cm}$ and depth $3=$ above $30 \mathrm{~cm}$ ) in 2013, 2015 and 2016 cropping seasons Soil moisture content at Bean harvesting

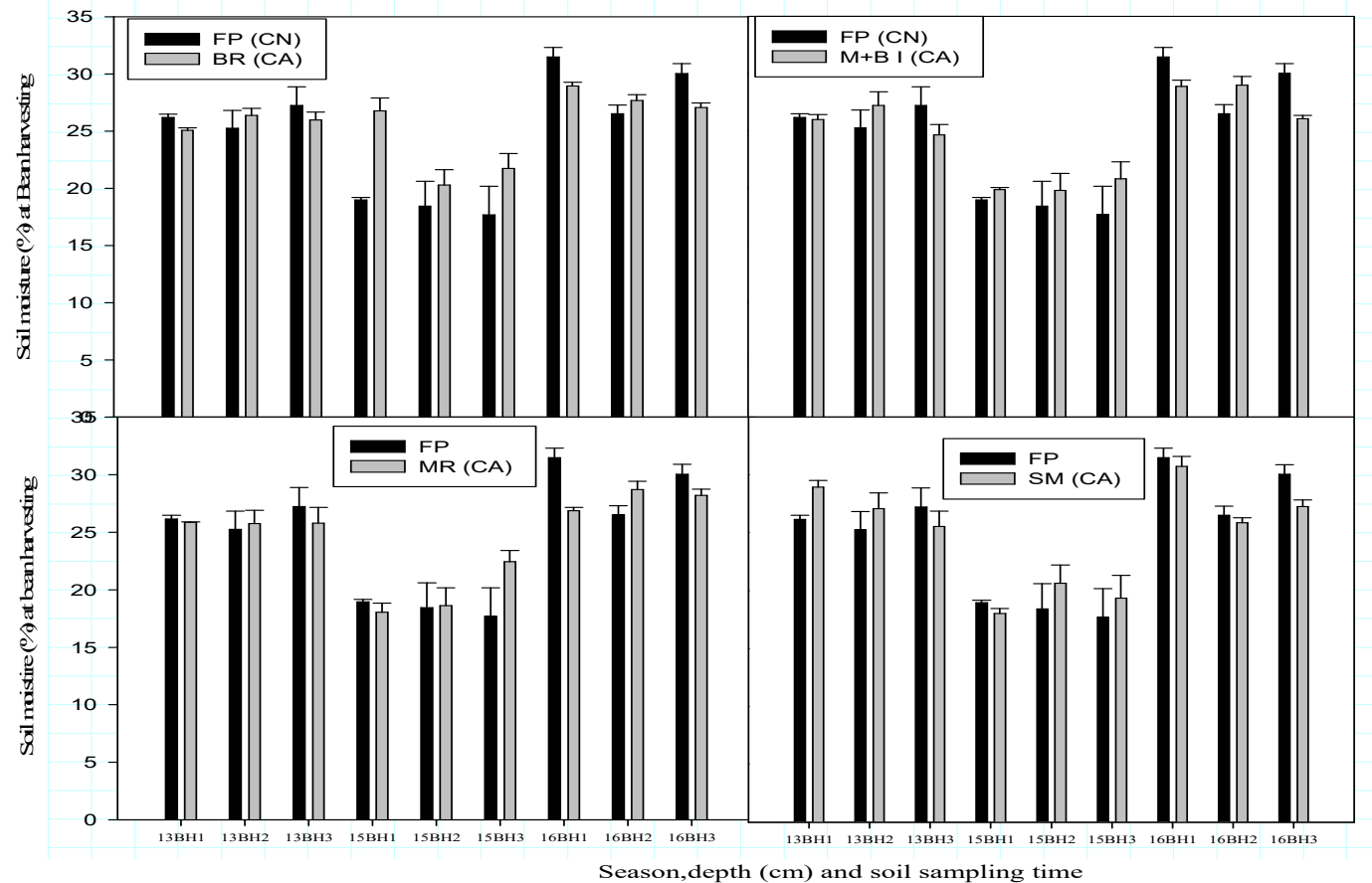

Fig.9. Soil moisture content in percentage at bean harvesting time at three soil depth (depth $1=0-15 \mathrm{~cm}$, depth $2=$ $15-30 \mathrm{~cm}$ and depth $3=$ above $30 \mathrm{~cm}$ ) in 2013, 2015 and 2016 cropping seasons 


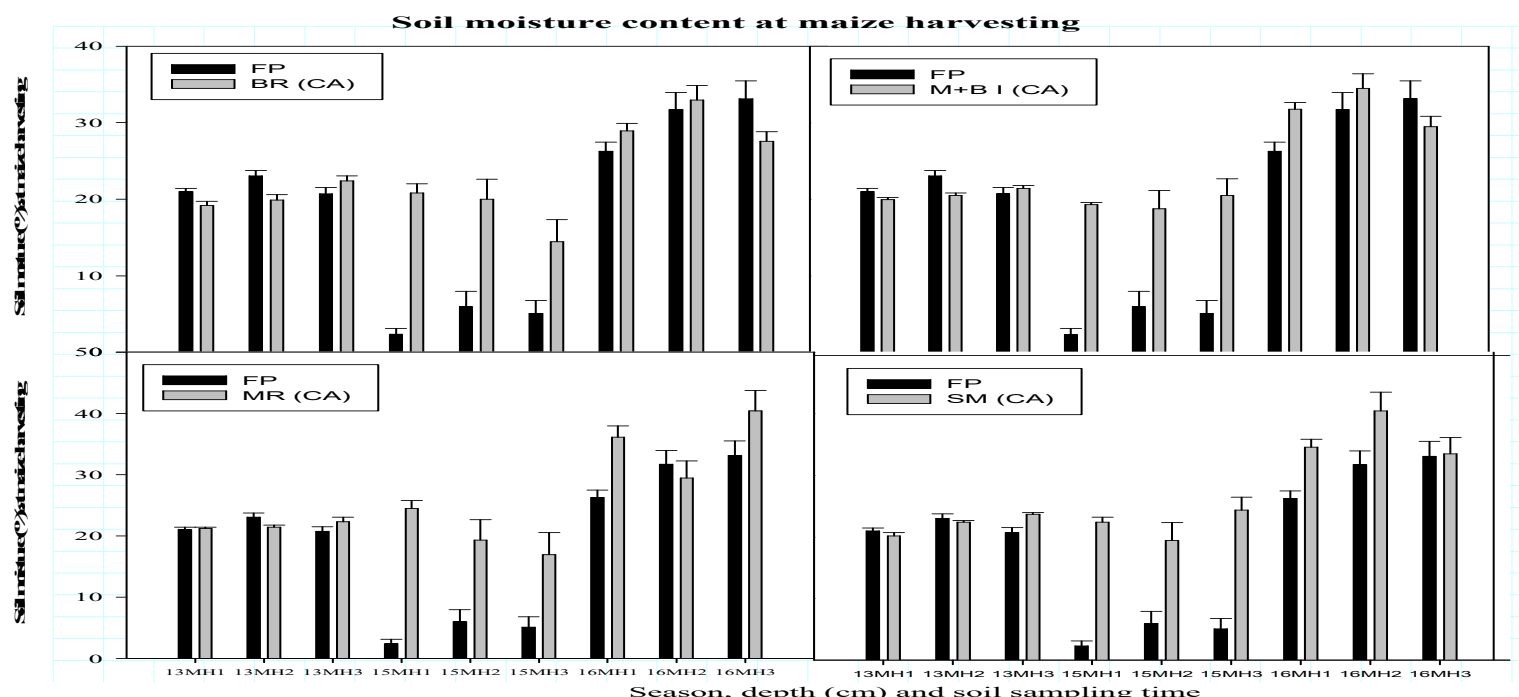

Fig.10. Soil moisture content in percentage at maize harvesting time at three soil depth (depth $1=0-15 \mathrm{~cm}$, depth $2=15-30 \mathrm{~cm}$ and depth $3=$ above $30 \mathrm{~cm}$ ) in 2013, 2015 and 2016 cropping seasons

Table 2 Soil moisture (\%) content at the three soil depths and in different cropping time within the season in 2013, 2015, and 2016 cropping season

\begin{tabular}{|c|c|c|c|c|c|}
\hline $\begin{array}{c}\text { Seaso } \\
\text { n }\end{array}$ & $\begin{array}{l}\text { Depth } \\
\text { (cm) }\end{array}$ & Treatment & $\begin{array}{c}\text { At } \\
\text { planting }\end{array}$ & $\begin{array}{c}\text { At bean } \\
\text { harvesting }\end{array}$ & $\begin{array}{c}\text { At maize } \\
\text { harvesting }\end{array}$ \\
\hline \multirow[t]{15}{*}{2013} & \multirow[t]{5}{*}{$0-15$} & Farmer Practice (sole maize) & $25 \mathrm{a}$ & $26 \mathrm{a}$ & $21 \mathrm{a}$ \\
\hline & & Bean Rotation (CA) & $23 \mathrm{a}$ & $25 \mathrm{a}$ & $19 \mathrm{a}$ \\
\hline & & $\mathrm{M}+\mathrm{B}$ Inter cropping $(\mathrm{CA})$ & $25 \mathrm{a}$ & $26 \mathrm{a}$ & $20 \mathrm{a}$ \\
\hline & & Maize Rotation (CA) & $25 \mathrm{a}$ & $26 \mathrm{a}$ & $21 \mathrm{a}$ \\
\hline & & Sole maize $(\mathrm{CA})$ & $24 \mathrm{a}$ & $29 \mathrm{a}$ & $20 \mathrm{a}$ \\
\hline & \multirow[t]{5}{*}{$15-30$} & Farmer Practice (sole maize) & $23 \mathrm{~b}$ & $25 \mathrm{a}$ & $23 a$ \\
\hline & & Bean Rotation (CA) & $22 b$ & $26 \mathrm{a}$ & $20 \mathrm{a}$ \\
\hline & & $\mathrm{M}+\mathrm{B}$ Inter cropping (CA) & $24 a b$ & $27 \mathrm{a}$ & $21 \mathrm{a}$ \\
\hline & & Maize Rotation (CA) & $27 \mathrm{a}$ & $26 \mathrm{a}$ & $21 \mathrm{a}$ \\
\hline & & Sole maize (CA) & $23 \mathrm{~b}$ & $27 \mathrm{a}$ & $22 \mathrm{a}$ \\
\hline & \multirow[t]{5}{*}{ above 30} & Farmer Practice (sole maize) & $18 \mathrm{~b}$ & $27 a$ & $21 b$ \\
\hline & & Bean Rotation (CA) & $21 \mathrm{ab}$ & $26 \mathrm{a}$ & $22 \mathrm{ab}$ \\
\hline & & $\mathrm{M}+\mathrm{B}$ Inter cropping (CA) & $26 \mathrm{a}$ & $25 \mathrm{a}$ & $21 \mathrm{ab}$ \\
\hline & & Maize Rotation (CA) & $22 \mathrm{ab}$ & $26 \mathrm{a}$ & $22 \mathrm{ab}$ \\
\hline & & Sole maize $(\mathrm{CA})$ & $18 \mathrm{ab}$ & $26 \mathrm{a}$ & $24 \mathrm{a}$ \\
\hline \multirow[t]{15}{*}{2015} & \multirow[t]{5}{*}{$0-15$} & Farmer Practice (sole maize) & $18 \mathrm{a}$ & $19 \mathrm{~b}$ & $16 \mathrm{a}$ \\
\hline & & Bean Rotation (CA) & $19 \mathrm{a}$ & $27 \mathrm{a}$ & $20 \mathrm{a}$ \\
\hline & & M + B Inter cropping (CA) & $22 \mathrm{a}$ & $20 \mathrm{~b}$ & $19 \mathrm{a}$ \\
\hline & & Maize Rotation (CA) & $21 \mathrm{a}$ & $18 \mathrm{~b}$ & $25 \mathrm{a}$ \\
\hline & & Sole maize $(\mathrm{CA})$ & $23 \mathrm{a}$ & $18 \mathrm{~b}$ & $23 \mathrm{a}$ \\
\hline & \multirow[t]{5}{*}{$15-30$} & Farmer Practice (sole maize) & $20 \mathrm{a}$ & $18 \mathrm{a}$ & $20 \mathrm{a}$ \\
\hline & & Bean Rotation (CA) & $21 \mathrm{a}$ & $20 \mathrm{a}$ & $19 \mathrm{a}$ \\
\hline & & $\mathrm{M}+\mathrm{B}$ Inter cropping (CA) & $20 \mathrm{a}$ & $20 \mathrm{a}$ & $19 \mathrm{a}$ \\
\hline & & Maize Rotation (CA) & $17 \mathrm{a}$ & $19 \mathrm{a}$ & $19 \mathrm{a}$ \\
\hline & & Sole maize $(\mathrm{CA})$ & $17 \mathrm{a}$ & $21 \mathrm{a}$ & $19 \mathrm{a}$ \\
\hline & \multirow[t]{5}{*}{ above 30} & Farmer Practice (sole maize) & $20 \mathrm{a}$ & $18 \mathrm{a}$ & $20 \mathrm{ab}$ \\
\hline & & Bean Rotation (CA) & $18 \mathrm{a}$ & $22 \mathrm{a}$ & $14 \mathrm{~b}$ \\
\hline & & M + B Inter cropping (CA) & $22 \mathrm{a}$ & $21 \mathrm{a}$ & $21 \mathrm{ab}$ \\
\hline & & Maize Rotation (CA) & $19 \mathrm{a}$ & $22 \mathrm{a}$ & $19 \mathrm{ab}$ \\
\hline & & Sole maize (CA) & $17 \mathrm{a}$ & $20 \mathrm{a}$ & $24 \mathrm{a}$ \\
\hline
\end{tabular}


Table 2 continued

\begin{tabular}{c|clccc}
\hline \multirow{3}{*}{ Season } & depth(cm) & Treatment & $\begin{array}{c}\text { At } \\
\text { planting }\end{array}$ & At bean harvesting & At maize harvesting \\
\hline 2016 & $0-15$ & Farmer Practice (sole maize) & $31 \mathrm{a}$ & $32 \mathrm{a}$ & $26 \mathrm{~b}$ \\
& & $27 \mathrm{a}$ & $29 \mathrm{a}$ & $29 \mathrm{ab}$ \\
& & Bean Rotation (CA) & $29 \mathrm{a}$ & $32 \mathrm{ab}$ \\
& M + B Inter cropping (CA) & $28 \mathrm{a}$ & $27 \mathrm{a}$ & $36 \mathrm{a}$ \\
& Maize Rotation (CA) & $32 \mathrm{a}$ & $31 \mathrm{a}$ & $35 \mathrm{ab}$ \\
\hline & Sole maize (CA) & $31 \mathrm{a}$ & $26 \mathrm{a}$ & $32 \mathrm{a}$ \\
& \multirow{2}{*}{$15-30$} & Farmer Practice (sole maize) & $26 \mathrm{a}$ & $28 \mathrm{a}$ & $33 \mathrm{a}$ \\
& & $29 \mathrm{a}$ & $29 \mathrm{a}$ & $30 \mathrm{a}$ \\
& & Bean Rotation (CA) & $25 \mathrm{a}$ & $29 \mathrm{a}$ & $31 \mathrm{a}$ \\
\cline { 3 - 5 } & M + B Inter cropping (CA) & $26 \mathrm{a}$ & $26 \mathrm{a}$ & $28 \mathrm{a}$ \\
& Maize Rotation (CA) & $26 \mathrm{a}$ & $30 \mathrm{a}$ & $30 \mathrm{a}$ \\
& Sole maize (CA) & $27 \mathrm{a}$ & $27 \mathrm{a}$ & $40 \mathrm{a}$ \\
& Farmer Practice (sole maize) & $26 \mathrm{a}$ & $26 \mathrm{a}$ & $34 \mathrm{a}$ \\
\hline
\end{tabular}

At East-Badawacho at planting, soil there was significant difference between treatments at $15-30 \mathrm{~cm}$ soil depth. The highest soil moisture was obtained on bean maize rotation. At soil depth of $>30 \mathrm{~cm}$ the difference was significant at planting time. At maize harvesting, the difference was significant at $0-15 \mathrm{~cm}$ soil depth and the highest soil moisture was obtained from sole maize under CA (Table 3). At Meskan, at planting, the variance was significant between treatments for soil moisture at bean harvesting at $0-15 \mathrm{~cm}$ soil depth. The highest soil moisture obtained from sole maize under CA. At soil depth $>30 \mathrm{~cm}$ the difference was significant between treatments at planting, bean harvesting and maize harvesting time. At planting time, at soil depth of $>30 \mathrm{~cm}$ the highest soil moisture value was obtained from bean maize rotation, at bean harvesting time, the highest value recorded FP sole maize whereas at maize harvesting, the highest value obtained from sole maize under CA similar soil depth (Table 3). At Hawassa Zuriya, at planting time the difference was significant between treatments $>30 \mathrm{~cm}$ soil depth with the highest value obtained from bean maize rotation. At bean harvesting, there was significant difference between treatments at soil depth of $0-15$ and $>30 \mathrm{~cm}$. The highest value was obtained from bean-maize rotation at $0-15 \mathrm{~cm}$ soil depth but soil depth $>30 \mathrm{~cm}$ the highest soil moisture was obtained from FP-sole maize. This indicated that CA practice had the chance to increase the water infiltration. Similarly, Kabirigi (2015) reported higher infiltration rate from not till practice with four different crop residue conditions (no till with: no input (control), inorganic fertilizer, residues, residue + inorganic fertilizer) compared with conventional practice with four residue conditions mentioned for no till. At maize harvesting time, the difference was significant between treatment at soil depth of $>$ $30 \mathrm{~cm}$ (Table 3). Similarly, Benites and Navarrete (2003) also reported as conservation agriculture is one way of improving soil moisture management trough combining the four principle of conservation agriculture (reducing sol disturbance, maintain permanent soil cover, controlling in field traffic and crop rotation). 
Table 3 Soil moisture (\%) content at the three soil depths and in different cropping time combined across three production seasons $(2013,2015$ and 2016) for each testing location

\begin{tabular}{|c|c|c|c|c|c|}
\hline location & $\operatorname{depth}(\mathrm{cm})$ & Treatment & Planting & Bean Harvesting & Maize Harvesting \\
\hline \multirow[t]{15}{*}{ East-Badawacho } & \multirow[t]{5}{*}{$0-15$} & FP-sole maize & $27 \mathrm{a}$ & $26 \mathrm{a}$ & $24 b$ \\
\hline & & HB-M Rotation (CA) & $23 \mathrm{a}$ & $27 \mathrm{a}$ & $28 \mathrm{ab}$ \\
\hline & & Inter cropping (CA) & $28 \mathrm{a}$ & $26 a$ & $26 \mathrm{ab}$ \\
\hline & & M_HB Rotation (CA) & $31 \mathrm{a}$ & $24 \mathrm{a}$ & $28 \mathrm{ab}$ \\
\hline & & Sole maize (CA) & $29 \mathrm{a}$ & $24 \mathrm{a}$ & $30 \mathrm{a}$ \\
\hline & \multirow[t]{5}{*}{$15-30$} & FP-sole maize & $20 b$ & $23 a$ & $26 a$ \\
\hline & & HB-M Rotation (CA) & $25 \mathrm{ab}$ & $23 a$ & $28 \mathrm{a}$ \\
\hline & & Inter cropping (CA) & $23 \mathrm{ab}$ & $24 \mathrm{a}$ & $27 \mathrm{a}$ \\
\hline & & M_HB Rotation (CA) & $27 \mathrm{a}$ & $25 a$ & $27 \mathrm{a}$ \\
\hline & & Sole maize $(\mathrm{CA})$ & $24 \mathrm{~b}$ & $24 \mathrm{a}$ & $32 \mathrm{a}$ \\
\hline & \multirow[t]{5}{*}{ above 30} & FP-sole maize & $20 \mathrm{ab}$ & $23 a$ & $26 a$ \\
\hline & & HB-M Rotation (CA) & $21 \mathrm{ab}$ & $26 a$ & $26 a$ \\
\hline & & Inter cropping (CA) & $23 \mathrm{a}$ & $25 \mathrm{a}$ & $26 \mathrm{a}$ \\
\hline & & M_HB Rotation (CA) & $22 \mathrm{a}$ & $27 \mathrm{a}$ & $31 \mathrm{a}$ \\
\hline & & Sole maize $(\mathrm{CA})$ & $18 \mathrm{~b}$ & $24 \mathrm{a}$ & $28 \mathrm{a}$ \\
\hline \multirow[t]{15}{*}{ Meskan } & \multirow[t]{5}{*}{$0-15$} & FP-sole maize & $25 \mathrm{a}$ & $27 \mathrm{ab}$ & $17 \mathrm{a}$ \\
\hline & & HB-M Rotation (CA) & $27 \mathrm{a}$ & $31 \mathrm{a}$ & $19 \mathrm{a}$ \\
\hline & & Inter cropping (CA) & $26 \mathrm{a}$ & $26 b$ & $21 \mathrm{a}$ \\
\hline & & M_HB Rotation (CA) & $26 \mathrm{a}$ & $25 b$ & $22 \mathrm{a}$ \\
\hline & & Sole maize $(\mathrm{CA})$ & $29 a$ & $28 \mathrm{ab}$ & $23 a$ \\
\hline & \multirow[t]{5}{*}{$15-30$} & FP-sole maize & $26 \mathrm{a}$ & $27 \mathrm{a}$ & $20 \mathrm{a}$ \\
\hline & & HB-M Rotation (CA) & $26 \mathrm{a}$ & $29 \mathrm{a}$ & $21 \mathrm{a}$ \\
\hline & & Inter cropping (CA) & $27 \mathrm{a}$ & $28 \mathrm{a}$ & $21 \mathrm{a}$ \\
\hline & & M_HB Rotation (CA) & $24 a$ & $27 \mathrm{a}$ & $21 \mathrm{a}$ \\
\hline & & Sole maize $(\mathrm{CA})$ & $25 \mathrm{a}$ & $25 \mathrm{a}$ & $20 \mathrm{a}$ \\
\hline & \multirow[t]{5}{*}{ above 30} & FP-sole maize & $24 \mathrm{ab}$ & $29 a$ & $23 \mathrm{ab}$ \\
\hline & & HB-M Rotation (CA) & $26 a b$ & $24 b$ & $17 \mathrm{~b}$ \\
\hline & & Inter cropping (CA) & $31 \mathrm{a}$ & $28 \mathrm{ab}$ & $24 \mathrm{ab}$ \\
\hline & & M_HB Rotation (CA) & $24 \mathrm{ab}$ & $28 \mathrm{ab}$ & $21 \mathrm{ab}$ \\
\hline & & Sole maize $(\mathrm{CA})$ & $21 \mathrm{~b}$ & $27 \mathrm{ab}$ & $30 \mathrm{a}$ \\
\hline
\end{tabular}

Table 3 continued

\begin{tabular}{cclccc}
\hline location & depth $(\mathrm{cm})$ & Treatment & Planting & Bean Harvesting & Maize Harvesting \\
\hline Hawassa-Zuriya & $0-15$ & FP-sole maize & $16 \mathrm{a}$ & $23 \mathrm{a}$ & $16 \mathrm{a}$ \\
& & HB-M Rotation $(\mathrm{CA})$ & $15 \mathrm{a}$ & $22 \mathrm{a}$ & $11 \mathrm{a}$ \\
& & $19 \mathrm{a}$ & $22 \mathrm{a}$ & $15 \mathrm{a}$ \\
& Inter cropping (CA) & $16 \mathrm{a}$ & $22 \mathrm{a}$ & $23 \mathrm{a}$ \\
& M_HB Rotation (CA) & $15 \mathrm{a}$ & $26 \mathrm{a}$ & $13 \mathrm{a}$ \\
\hline \multirow{2}{*}{$15-30$} & Sole maize (CA) & FP-sole maize & $20 \mathrm{a}$ & $20 \mathrm{~b}$ & $22 \mathrm{a}$ \\
& & HB-M Rotation (CA) & $16 \mathrm{ab}$ & $23 \mathrm{ab}$ & $13 \mathrm{a}$ \\
& Inter cropping (CA) & $16 \mathrm{ab}$ & $24 \mathrm{ab}$ & $15 \mathrm{a}$ \\
& M_HB Rotation (CA) & $16 \mathrm{ab}$ & $21 \mathrm{ab}$ & $15 \mathrm{a}$ \\
& Sole maize (CA) & $12 \mathrm{~b}$ & $25 \mathrm{a}$ & $16 \mathrm{a}$ \\
\cline { 3 - 5 } & above 30 & FP-sole maize & $18 \mathrm{a}$ & $23 \mathrm{ab}$ & $16 \mathrm{a}$ \\
& HB-M Rotation (CA) & $13 \mathrm{a}$ & $25 \mathrm{a}$ & $13 \mathrm{a}$ \\
& Inter cropping (CA) & $18 \mathrm{a}$ & $18 \mathrm{~b}$ & $14 \mathrm{a}$ \\
& M_HB Rotation (CA) & $18 \mathrm{a}$ & $21 \mathrm{ab}$ & $15 \mathrm{a}$ \\
& Sole maize (CA) & $18 \mathrm{a}$ & $23 \mathrm{ab}$ & $16 \mathrm{a}$ \\
\hline
\end{tabular}

\subsection{NDVI}

There was significant difference among treatments for NDVI with the highest NDVI observed for rotation and sole maize under CA compared with farmers practice (Table 4). Higher NDVI values for CA than CN at vegetative and flowering reflected higher growth for CA treatments than CN (Table 4) (Verhulst et al. 2011). This was because drought stress conditions enhanced earlier reduction of the NDVI values (Verhulst et al. 2011). Except for at famer 3, CA treatments have significantly higher NDVI values compared with farmers' practice (Fig. 8). 
Table 4. Mean square and mean of NDVI for five treatments tested at three districts in the year 2016 cropping season at East-Badawacho.

\begin{tabular}{|c|c|c|c|c|}
\hline $\begin{array}{l}\text { Source } \\
\text { Variation }\end{array}$ & $\begin{array}{ll}\text { of } & \mathrm{D} \\
& \mathrm{F} \\
\end{array}$ & $\begin{array}{l}\text { Mean } \\
\text { Square }\end{array}$ & Treatment & $\begin{array}{l}\text { Mean of leaf greenness (chlorophyll) } \\
\text { (NDVI) }\end{array}$ \\
\hline Farmer/rep & 4 & 0.004 & Farmer practice $(\mathrm{CN})$ & $0.58 b$ \\
\hline Treatment & 2 & $0.04 * *$ & Sole Maize (CA) & $0.74 \mathrm{a}$ \\
\hline error & 8 & 0.004 & Maize Rotation (CA) & $0.73 a$ \\
\hline $\mathrm{cV}$ & & 8.93 & & \\
\hline mean & & 0.68 & & \\
\hline LSD & & & & 0.09 \\
\hline
\end{tabular}

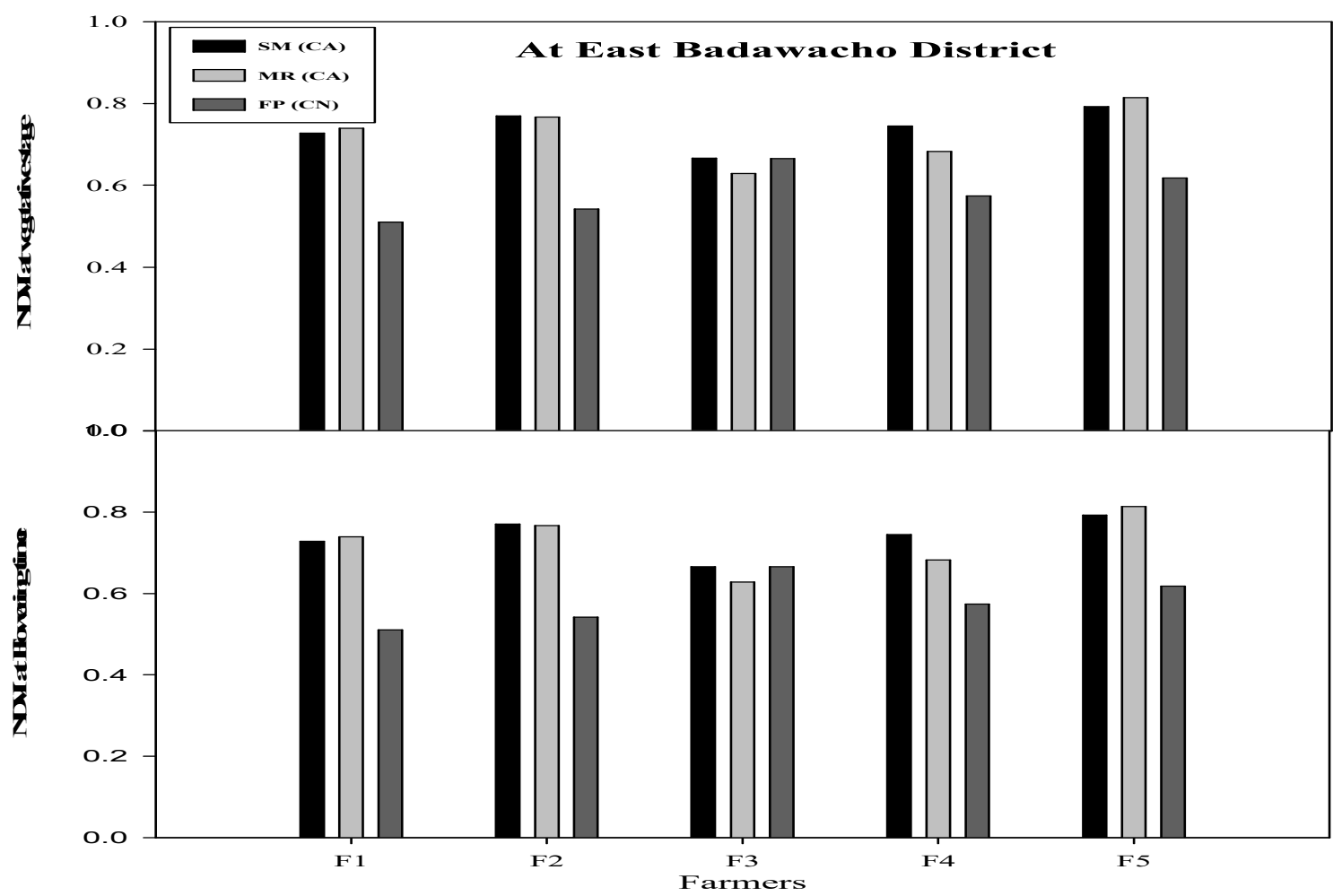

Fig.11. Maize leaf chlorophyll content result during 2016 cropping season from CA and farmer practice 


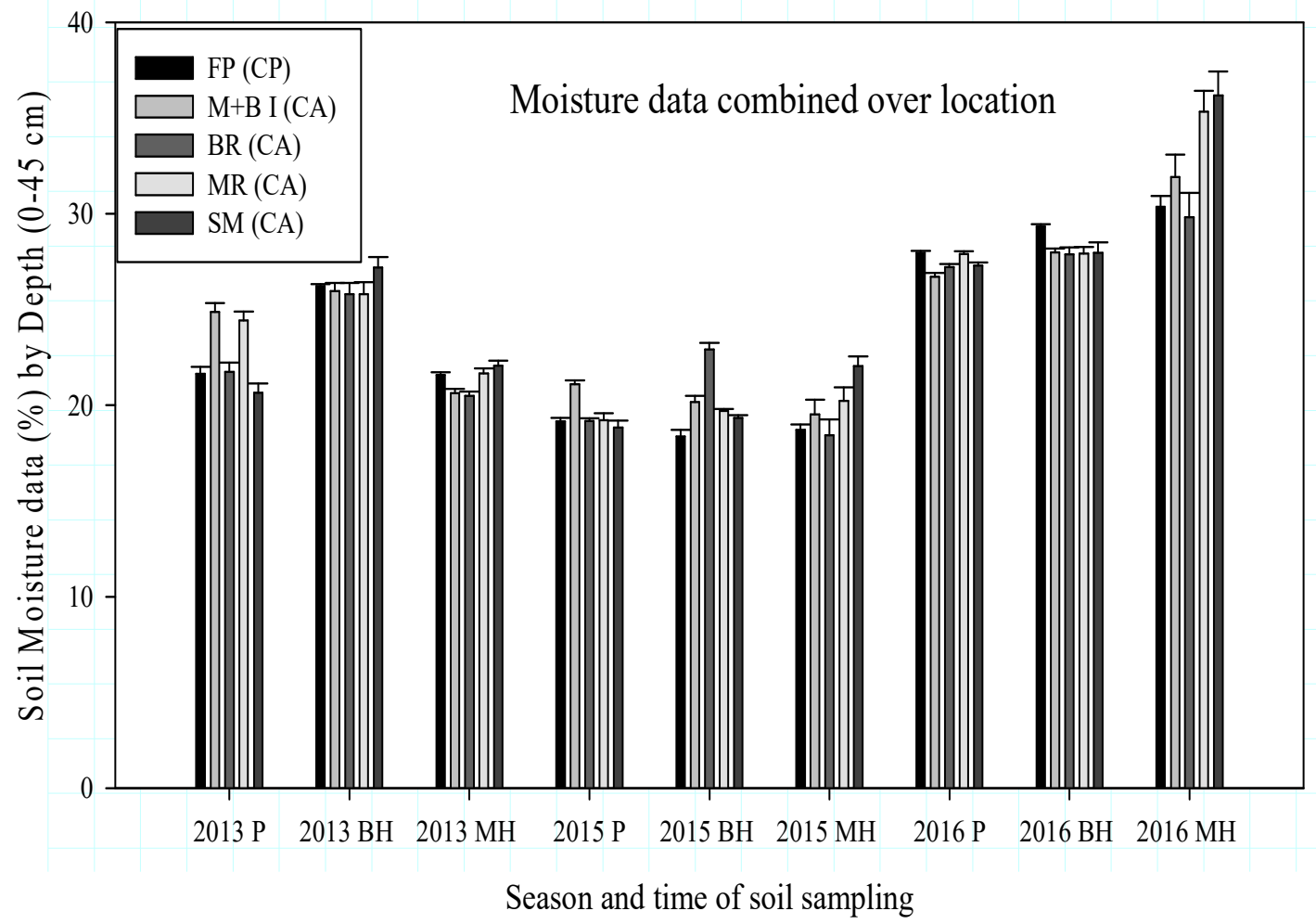

Fig.12. Soil moisture content at soil depth of 0-45 $\mathrm{cm}$ from combined data over season and location

\subsection{Grain and biomass yield}

At three locations the variance between treatments was significant for biomass. The significant difference for variance of treatment*season for biomass and grain yield indicates that the treatments were responded differently at different cropping season (Table 5).

Table 5: Analysis of variance between treatments for combined analysis across season

\begin{tabular}{|c|c|c|c|c|}
\hline Location & $\begin{array}{l}\text { Source of } \\
\text { Variation }\end{array}$ & DF & Biomass & Grain Yield \\
\hline \multirow{7}{*}{ East-Badawacho } & Rep/farmer & 4 & $144813510 * * *$ & $18911849 * * *$ \\
\hline & Treatment & 3 & $42324005^{*}$ & 2690405 \\
\hline & Treatment*season & 20 & $54705402 * * *$ & $3150452 *$ \\
\hline & Error & 96 & 11974474 & 1648905 \\
\hline & $\mathrm{CV}$ & & 29.5 & 32.6 \\
\hline & Mean & & 11712 & 3940 \\
\hline & LSD & & 1806 & 659 \\
\hline \multirow{7}{*}{ Meskan } & Rep/farmer & 4 & 15597929 & $1990395^{*}$ \\
\hline & Treatment & 3 & 2412469 & 412011 \\
\hline & Treatment*season & 20 & $35174758 * *$ & $9513777 * * *$ \\
\hline & Error & & 13101393 & 910562 \\
\hline & $\mathrm{CV}$ & & 36.4 & 27.7 \\
\hline & Mean & & 9945 & 3447 \\
\hline & LSD & & 2224 & 858 \\
\hline \multirow{7}{*}{ Hawassa-Zuriya } & Rep/farmer & & 16742667 & $11240836^{*}$ \\
\hline & Treatment & & 14028729 & 7733831 \\
\hline & Treatment*season & & $54449595 * * *$ & $7272154^{*}$ \\
\hline & Error & & 8875894 & 4002536 \\
\hline & $\mathrm{CV}$ & & 31.6 & 27.7 \\
\hline & Mean & & 9430 & 4421 \\
\hline & LSD & & 2087 & 1004 \\
\hline
\end{tabular}




\subsection{Grain yield}

For grain yield, six-year average data and mean from cropping systems showed that CA practice had higher yield performance than FP by 4\% at East-Badawacho. while considering six-year average by each cropping system, maize rotation and sole maize under CA had higher grain yield advantage over FP by 13 and 4\%, respectively but maize-bean inter cropping under CA had inferior yield performance by $5 \%$ compared with FP without considering common bean grain yield obtained from inter-cropping. When the output of bean from inter cropping added, intercropping had remarkable potential compared with the farmer practice and other cropping systems under CA (Table 6). similarly, Yilmaz et al. (2008) reported higher yield advantage from $67 \%$ maize mix with $50 \%$ bean or cowpea in both 1 maize: 1 bean and 2 maize: 2 bean or in one row and two row planting patter compared to solitary cropping of the same species.

Under each season consideration, inter-cropping under CA had 4\% advantage than FP for maize grain yield during the worst season (2012). The reason may be due to the space between maize rows covered by common bean which help to protect soil moisture from evaporation and make it available for maize and common bean crops. During the remaining five years (relatively good season compared with 2012), maize-bean inter cropping system had somehow inferior performance for maize grain yield than FP without considering the grain yield advantage obtained from common bean. Similarly, Cameron et al. (2014) reported significantly enhanced yields (7\%) under rain fed agriculture from no till in dry Climates when the other two conservation agriculture principles are also implemented. Cameron also reported the reverse result that is when no-till is applied alone the yield reduced by $12 \%$. Maize rotation under CA had higher grain yield advantage than FP by 25, 15, 5, 26 and 20\% in 2012, 2013, 2014, 2015 and 2016, respectively except, the first season (2011) in which maize rotation under CA had lower grain yield advantage than FP by $1 \%$. Comparing sole maize under CA, it had higher grain yield advantage than FP by 15, 7, 11 and 16\% in 2012, 2013, 2014 and 2015, respectively but during starting year (2011) and last year (2016) of the experimentation, the performance of sole maize under CA had lower performance than FP. At Hawassa-Zuriya, Maize rotation under CA out yielded than FP in 2011 and 2013 by 19\% and 2\%, respectively. Whereas sole maize under CA had 4\% yield advantage during first year (2011) experiment. Thierfelder et al. (2012) also reported higher benefits of crop rotation over continuous maize and intercropped maize.

Six-year combined analysis result showed that cropping practices under CA had lower performance compared with farmer practice (Table 6) which in line with the report of Cameron et al. (2014) reported overall reduced crop yields (by 6\%) from no-till and also reported variable response under certain conditions meaning no-till can also produce equivalent or greater yields than conventional tillage based on global meta-analysis using 5,463 paired yield observations from 610 studies to compare no-till, the original and central concept of conservation agriculture, with conventional tillage practices across 48 crops and 63 countries. Cameron et al. (2014) also told us as no-till is combined with the other two conservation agriculture principles of residue retention and crop rotation, its negative impacts are minimized and significantly increases rain fed crop productivity in dry climates, suggesting that the combination of the three CA components may become an important climate-change adaptation strategy forever-drier regions of the world. At Meskan, six-year average data of each cropping systems: sole maize, maize rotation and maize-bean inter cropping under CA had higher grain yield compared to FP with the value of 10,8 and $6 \%$, respectively (Table 6). For individual season and each cropping system consideration, inter cropping under CA had higher grain yield advantage than FP in 2011, 2012, 2013 and 2014 with the value of 0.2, 86, 37 and $8 \%$, respectively. Maize rotation under CA also had higher grain yield performance during 2012, 2013 and 2016 with magnitude of the value was 109,68 and 4\%, respectively. Sole maize under CA had also superior grain yield performance than FP in 2011, 2012, 2013 and 2016 with the magnitude of $0.2,71,59$, and 2\%, respectively. The variation for the performance of cropping systems was due to the seasonal variability. At Meskan, across sixyear and cropping systems, mean grain yield result showed that the performance of CA practices had higher value than FP by $8 \%$. The combined data from East-Badawacho and Meskan also showed that CA had higher grain yield advantage than FP by the value of $6 \%$. The higher grain and biomass yield obtained from CA indicated that under CA crop had better water use efficiency compared with FP. Similarly, Araya et al. (2012) recorded high water use efficiency in permanent raised beds (Conservation tillage) with $30 \%$ standing crop residue retention compared to treatments ploughed once at sowing with 30\% standing crop residue retention and conventional tillage. The survey results of Musa (2014) on determinant factors for adoption of crop rotation in Arsi-Negele, Ethiopia: indicated that, regular education, farming experience (number of years the farmer spent in the agriculture) and frequency of contacts with extension workers in a year had significant contribution for adoption of the practice. Generally, any expansion of conservation agriculture should be done with caution in drier areas, as implementation of the other two principles (residue retention and crop rotation) is often challenging in resource-poor and vulnerable smallholder farming systems, thereby increasing the likelihood of yield losses rather than gains. A yield benefit with no-till in combination with the other two conservation agriculture principles in dry climates is probably because of improved water infiltration and greater soil moisture conservation (Serraj and Siddique 2012). This finding suggests that if no-till applied in combination with the other two conservation agriculture principles, can become an increasingly important strategy to deal with soil moisture stress due to climate change. It is precisely 
resource-poor and vulnerable smallholder farming systems that will have the greatest challenges adopting the other two principles, most notably the retention of crop residues due to strong competition for residues by livestock and other uses (Giller et al. 2009; Erenstein et al. 2012). The comparative productivity analysis between continuous maize, maize intercropped with cowpea or pigeonpea and maize in rotation with cowpea or sunnhemp, shows marked benefits of rotation especially in CA systems (Thierfelder et al. 2012). Kabirigi (2015) also reported higher maize grain yield under CA practices compared with the maize grain yield from conventional practice.

\subsection{Biomass yield}

The across season and cropping systems analysis, biomass yield advantage form CA was $21 \%$ compared with FP at East-Badawacho (Table 6). In the across season data analysis, under each cropping system under CA (maizebean inter cropping, maize rotation and sole maize) had higher biomass yield than FP by the magnitude of 14, 27, and 22\%, respectively. During each season, each cropping system under CA (maize-bean inter-cropping under CA had higher performance than FP in 2012, 2014, 2015 and 2016 with magnitude of 4, 30, 24, and 52\%, respectively. Maize rotation under CA had higher biomass yield than FP with the value of 14, 17, 31, 77 and 42\% in 2012, 2013, 2014, 2015 and 2016, respectively except in 2011 (first experiment year). While considering the relative performance of sole maize under CA, it had higher biomass yield than FP with the value of 4 (in 2011), 3 (2012), 29 (2013), 30 (2014), 64 (2015) and 17\% (2016). Generally, the higher maize grain and biomass yield in 2016 evidence supported by availability of high chlorophyll content in maize leaf at vegetative and flowering stage of the crop compared with FP (Fig. 11). At Hawassa-Zuriya, maize-bean inter-cropping had higher biomass yield performance than FP in 2011 and 2016 by 11, and 2\%, respectively. Maize rotation exhibited higher biomass yield in 2011, 2013 and 2016 with the magnitude of 42, 2 and 7\%, respectively. Sole maize under CA also had higher biomass yield with the value of $53 \%$ in 2011 cropping season but during the rest of the cropping season, this treatment had inferior performance as compared with FP. At Meskan, the overall performance cropping systems under CA had higher biomass yield than FP by $8 \%$ biomass advantage which is computed form combined across season and cropping systems. Kabirigi (2015) reported significantly higher stover yield from CA practices compared with the conventional practices.

From six-year combined data analysis, maize-bean inter-cropping, maize rotation and sole maize under CA had higher biomass yield over FP by 9,4 , and 10\%, respectively. while considering each cropping systems at each season, maize-bean inter-cropping under CA had higher biomass yield than FP in 2011, 2013 and 2016 with the magnitude of 20, 53, and 56\%, respectively. Maize rotation also had higher biomass yield than FP in 2011, 2013 and 2016 with value of 21, 41, and 26\%, respectively. Similarly, sole maize under CA had higher biomass yield than FP in 2011, 2013 and 2016 with magnitude of 11, 55, and 83\%, respectively.

Biomass yield across season and location: from data combined over season and location: Maize-bean intercropping, maize rotation and sole maize under CA had higher biomass advantage over FP by 1, 8, and $8 \%$, respectively. For each cropping system during each season combined across location, maize-bean inter-cropping had higher biomass yield in 2011 and 2016 with magnitude of 6 and 36\%, respectively but during the other seasons, this treatment had inferior performance than FP. Maize rotation under CA also had relative higher biomass advantage than FP in 2011, 20132015 and 2016 with magnitude of 22, 14, 14, and 25\% respectively. Similarly, Thierfelder et al. (2012) reported higher biomass production from maize rotation compared to continuous sole maize from research conducted in Zimbabwe under CA for six years. Sole maize under CA had remarkable performance for biomass yield over FP in 2011, 2013, 2015 and 2016 with magnitude of 28, 20, 14, and 22\%, respectively. The overall performance of CA was higher by $6 \%$ for biomass compared with FP based the average data from across six-year and locations analysis. The increase in grain and biomass yield in no tillage is in contrast with the finding by Mehmood et al. (2014) who reported under zero tillage wheat straw mulch have inferior yield than conventional tillage. 
Table 6 Mean performance of CA and CP treatment over season (2011-2016) at three locations

\begin{tabular}{llcc}
\hline Location & Treatments & $\begin{array}{c}\text { Biomass } \\
\left(\mathrm{kg} \mathrm{ha}^{-1}\right)\end{array}$ & $\begin{array}{c}\text { Grain yield } \\
\left(\mathrm{Kg} \mathrm{ha}^{-1}\right)\end{array}$ \\
\hline East-Badawacho & Farmer Practice (sole maize) & $10100 \mathrm{~b}$ & $3829 \mathrm{ab}$ \\
& Sole Maize CA & $12365 \mathrm{a}$ & $3965 \mathrm{ab}$ \\
& Maize Rotation CA & $12859 \mathrm{a}$ & $4338 \mathrm{a}$ \\
& M + B Inter-cropping & $11523 \mathrm{ab}$ & $3628 \mathrm{~b}$ \\
& F-test & $*$ & Ns \\
& CV (\%) & 29.5 & 32.6 \\
\hline Feskan & Farmer Practice (sole maize) & $10502 \mathrm{a}$ & $3227 \mathrm{a}$ \\
& Sole Maize CA & $9593 \mathrm{a}$ & $3554 \mathrm{a}$ \\
& Maize Rotation CA & $9723 \mathrm{a}$ & $3494 \mathrm{a}$ \\
& M + B Inter-cropping & $9842 \mathrm{a}$ & $3421 \mathrm{a}$ \\
& F-test & $\mathbf{n s}$ & 27.7 \\
\hline CV (\%) & 36.4 & $5606 \mathrm{a}$ \\
& Farmer Practice (sole maize) & $10932 \mathrm{a}$ & $4694 \mathrm{a}$ \\
& Sole Maize CA & $10022 \mathrm{a}$ & $4749 \mathrm{a}$ \\
& Maize Rotation CA & $9992 \mathrm{a}$ & $4325 \mathrm{a}$ \\
& M B Inter-cropping & $8776 \mathrm{a}$ & Ns \\
& F-test & ns & 27.7 \\
\hline
\end{tabular}

\subsection{Common bean performance}

Regarding the common bean data, for bean rotation the mean performance from across season and location was $2978 \mathrm{~kg} \mathrm{ha}^{-1}$ for grain yield and for inter cropping the mean value was $935 \mathrm{~kg} \mathrm{ha}^{-1}$. The grain yield and biomass production from inter cropping is the additional produce on yield of maize for grain and biomass. The combined mean data across location and season also showed that, the biomass yield of bean from bean rotation and inter cropping was 5045 and $1658 \mathrm{~kg} \mathrm{ha}^{-1}$, respectively (Table 7). While considering the common bean production performance for grain yield and biomass, relatively higher magnitude was obtained from Meskan from overall consideration (Fig.13). Based on sample data, bean rotation had higher performance than inter cropping both under CA practice for HI, PPP, TSW and PH (Table 7).

Table 7 Mean performance of common bean combined data across (season and location) 2011-2016 under CA.

\begin{tabular}{lccccccccc}
\hline Treatments & $\begin{array}{c}\text { Biomass } \\
\left(\mathrm{Kg} / \mathrm{ha}^{-1}\right)\end{array}$ & $\begin{array}{c}\text { Grain } \\
\text { yield } \\
\left(\mathrm{kg} \mathrm{ha}^{-1}\right)\end{array}$ & $\begin{array}{c}\mathrm{HI} \\
(\%)\end{array}$ & $\begin{array}{c}\text { PPP } \\
(\#)\end{array}$ & $\begin{array}{c}\text { SPP } \\
(\#)\end{array}$ & $\begin{array}{c}\text { TSW } \\
(\mathrm{gm})\end{array}$ & $\begin{array}{c}\text { SHAV } \\
\# / \mathrm{ha}^{-1}\end{array}$ & $\begin{array}{c}\mathrm{NP} / \mathrm{m} 2 \\
(\#)\end{array}$ & $\begin{array}{c}\mathrm{PH} \\
(\mathrm{cm})\end{array}$ \\
\hline Bean rotation (CA) & $5045 \mathrm{a}$ & $2978 \mathrm{a}$ & $59 \mathrm{a}$ & $19 \mathrm{a}$ & $6 \mathrm{a}$ & $257 \mathrm{a}$ & $1648 \mathrm{a}$ & $17 \mathrm{a}$ & $50 \mathrm{a}$ \\
Inter cropping (CA) & $1658 \mathrm{~b}$ & $935 \mathrm{~b}$ & $56 \mathrm{a}$ & $13 \mathrm{~b}$ & $5 \mathrm{~b}$ & $254 \mathrm{a}$ & $788 \mathrm{~b}$ & $8 \mathrm{~b}$ & $44.6 \mathrm{~b}$ \\
CV (\%) & 34.2 & 32.2 & 30.5 & 35.4 & 12.7 & 17.5 & 21.4 & 21.4 & $\begin{array}{c}20.3 \\
* * *\end{array}$ \\
F-test & $* * *$ & $* * *$ & $\mathrm{~ns}$ & $* * *$ & $* * *$ & $\mathrm{~ns}$ & $* * *$ & $* * *$ & $* *$ \\
\hline
\end{tabular}

Where, $H I=$ harvest index in $\%, P P P=$ pod per plant (\#), SPP= seed per pod (\#), SHAV= stand count at harvest (\#), NP/m2= number of plants per meter square (\#), $P H=$ plant height $(\mathrm{cm})$ 

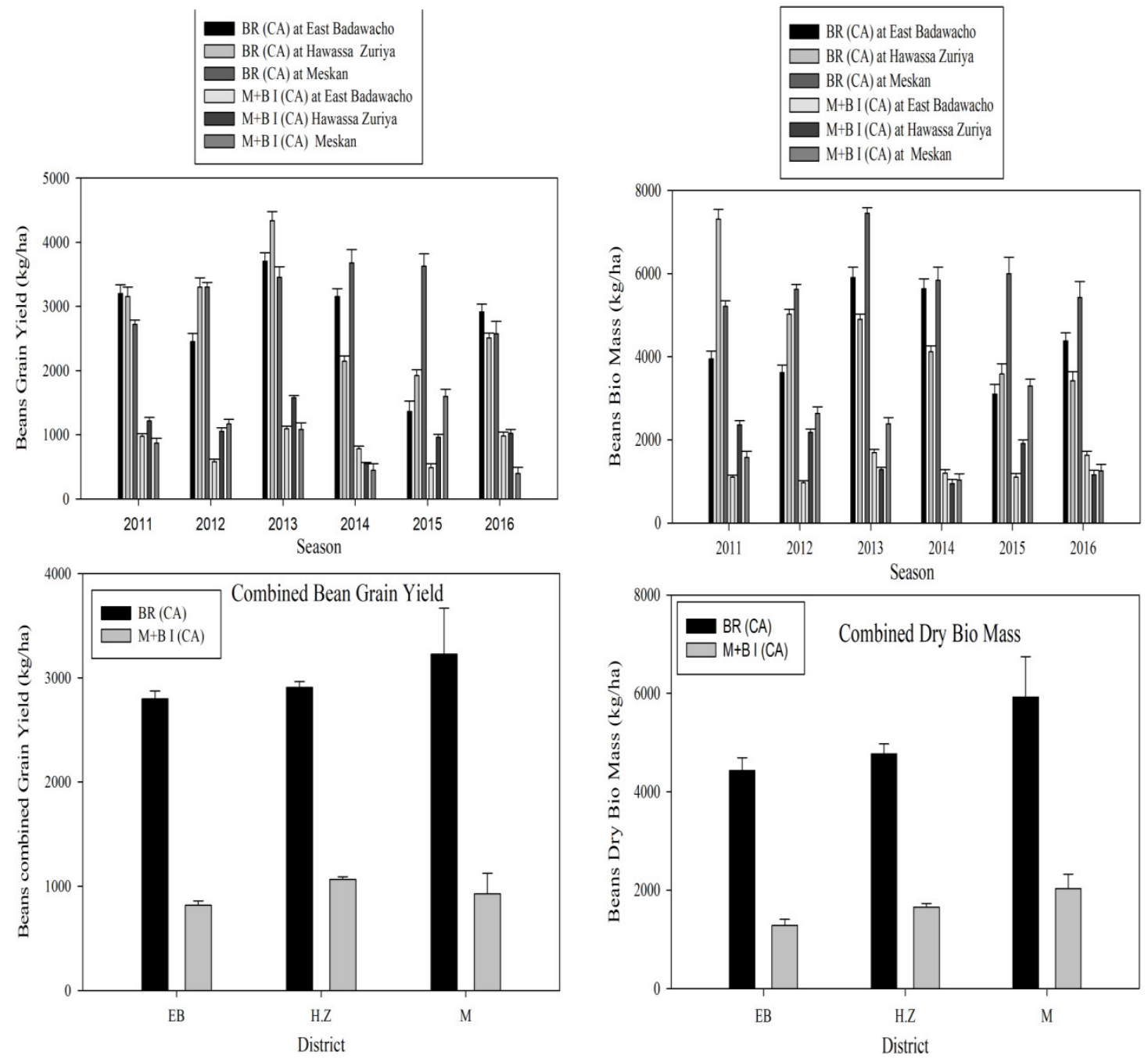

Fig.13. Biomass and grain yield mean performance for each location at each season and mean across season at each location

\section{Conclusion}

The overall assessment of cropping systems under CA and FP indicated that, cropping systems under CA performed better under CA than farmer practice both under normal and bad environmental condition for the production of grain yield and biomass. Soil moisture content from CA practices was higher than that of famer practices in general. When there was rain fall shortage, the performance of CA practices was better than that of common practice for production of higher grain yield and biomass due CA practices potential in reducing risk factor like low soil moisture content. During the presence of rainfall shortage, maize-bean inter cropping had potential in reducing the evaporation of soil moisture or conserving soil moisture as compared with the rest of cropping systems under CA and farmer practice. Considering production from maize crop only, maze rotation had relatively higher maize grain yield and biomass production potential as compared with others but while considering the merit in reduction of environmental risks and having addition yield from common bean, maize-bean inter cropping should be the best choose.

\section{Acknowledgment}

This research was funded by ACIAR (Australian Centre for International Agricultural Research) through Sustainable Intensification of Maize-Legume Cropping Systems for Food Security in Eastern and Southern Africa (SIMLESA) project. We thank Mrs. Maedin Tadesse, Mr. Zerihun Beshir, and Mr. Temesgen Gizaw for their assistance in data collection.

\section{Reference}

Addise, E. (2014), "The challenges and prospects of land restoration practices: the case of Misirak Badawacho woreda of Hadiya Zone, SNNPR, Ethiopia", MA. Thesis. Addis Ababa, Ethiopia. 
Agegnehu, G., Ghizam, A. \& Sinebo, W. (2006), "Yield performance and land-use efficiency of barley and faba bean mixed cropping in Ethiopian highlands", Eur. J. Agron. 25: 202-207.

Ananthi, T., Amanullah, M.M., Al-Tawaha, A. R. M. S. (2017), “A Review on Maize- Legume Inter-cropping For Enhancing the Productivity and Soil Fertility for Sustainable Agriculture in India", Advances in Environmental Biolograin yield, 11(5) May 2017, Pages: 49-63.

Anil, L., Park, J., Phipps, R.H. \& Miller F.A. (1998), “Temperate inter-cropping of cereals for forage: a review of the potential for growth and utilization with particular reference to the UK", Grass For. Sci. 53: 301-317.

Araya, T., Cornelis, W. M., Nyssen, J., Govaerts, B., Getnet, F., Bauer, H., Amare, K., Raes, D., Haile, M. \& Deckers, J. (2012), "Medium-term effects of conservation agriculture based cropping systems for sustainable soil and water management and crop productivity in the Ethiopian highlands", Field crops research, 132, 5362.

Banik, P., Midya, A., Sarkar, B.K. \& Ghose, S.S. (2006), "Wheat and chickpea inter-cropping systems in an additive series experiment: advantages and weed smothering", Eur. J. Agron. 24: 325-332.

Bayer, C., Mielniczuk,,J., Amado, T.J.C., Martin-Neto, L.\& Fernandes, S.V.(2000), "Organic matter storage in a sandy loam Acrisol affected by tillage and cropping systems in southern Brazil”, Soil Tillage Res. 54, 101109. (doi:10. 1016/S0167-1987(00)00090-8).

Benites, J. \& Navarrete, A.C. (2003), "Improving soil moisture with conservation agriculture. LEISA MAGAZINE".

Bissett, M. J. \& O’Leary, G.J. (1996), "Effects of conservation tillage on water infiltration in two soils in southeastern Australia”, Aust. J. Soil Res. 34, 299-308. (doi:10.1071/SR9960299).

Cameron, M. Pittelkow, Liang, X., Bruce, A., Linquist, Groenigen, K.J.V., Lee, J., Mark, E., Lundy, Gestel, N.V., Johan Six, Rodney, T., Venterea \& Kessel, C. V. (2014), Productivity limits and potentials of the principles of conservation agriculture", 2/Nature/Vol 000/00.

Chen, C., Westcott, M., Neill, K., Wichman D. \& Knox, M. (2004), "Row configuration and nitrogen application for barley-pea inter-cropping in Montana", Agron. J. 96: 1730-1738.

Dhima, K.V., Lithourgidis, A.A., Vasilakoglou, I.B. \& Dordas, C.A. (2007), "Competition indices of common vetch and cereal intercrops in two seeding ratio", Field Crop Res. 100: 249-256.

EOSA (Ethio-Organic Seed Action). (2007), "Baseline Survey of Dendi, Awassa Zuriya and Hetosa Districts of Ethiopia, Ethio-organic seed action in collaboration with center for genetic resources", the Netherland.

Erenstein, O., Sayre, K., Wall, P., Hellin, J.\& Dixon, J. (2012), "Conservation agriculture in maize- and wheatbased systems in the (sub)tropics: lessons from adaptation initiatives in South Asia", Mexico, and Southern Africa. J. Sustain. Agric.36, 180-206.

Farooq, M., \& Siddique, K.H.M. (2015), "Conservation Agriculture: Concepts, Brief History, and Impact on Agricultural System",https//www.researchgate.net/publication/270274873.

Fenandez-Aparicio, M., Josefina, C. \& Sillero, D.R. (2007), "Inter-cropping with cereals reduces infection by Orobanche crenata in legumes", Crop Protec. 26: 1166-1172.

Fitsum, S. (2002), "Contribution of fanyajuu and normal bund for crop production in Ethiopia", Addis Ababa, Ethiopia.83-84.

Food and Agriculture Organization (FAO). (2011), "Save and Grow: A Policymaker's Guide to the Sustainable Intensification of Smallholder Crop Production"” pp 1-37.

Friedrich, T., Derpsch, R.\& Kassam, A. (2012), Overview of the global spread of conservation agriculture", Field Actions Sci. Rep. 6, 1941.

Geremaw, S. (2005), Improve soil and water conservation on farm land on high land of Ethiopia", Addis Ababa, Ethiopia, 83-84.

Getahun, H., Mulugeta, L., Fisseha, I. \& Feyera S. (2014), "Impacts of Land Uses Changes on Soil Fertility, Carbon and Nitrogen Stock under Smallholder Farmers in Central Highlands of Ethiopia: Implication for Sustainable Agricultural Landscape Management Around Butajira Area", New York Science Journal 2014;7(2) http://www.sciencepub.net/newyork.

Giller, K. E., Witter, E., Corbeels M. \& Tittonell, P. (2009), “Conservation agriculture and smallholder farming in Africa: the heretics' view", Field Crops Res. 114, 23-34.

Govaerts, B., Verhulst, N., Sayre, K.D., Corte, P.D., Goudeseune, B., Lichter, K., Crossa, J., Deckers, J., Dendooven, L. (2007), "Evaluating spatial within plot crop variability for different management practices with an optical sensor", Plant Soil. 299, 29-42.

Hatfield, K.L. \& Pruegar, J.H. (1996), "Microclimate effects of crop residues on biological processes", Theor. Appl. Climatol. 54, 47-59. (doi:10.1007/BF00863558).

Herweg, LE. (1999), "The performance of selected soil and water conservation measures case studies from Ethiopia and Eritrea", Addis Ababa Ethiopia catena.36:99-114.

Hobbs, P. R., Sayre, K.\& Gupta. R. (2008), "The role of conservation agriculture in sustainable agriculture”, Phil. Trans. R. Soc. B 363, 543-555, doi:10.1098/rstb.2007.2169. 
Howard, R. J. (1996) “Cultural control of plant diseases: ahistorical perspective”, Can. J. Plant Pathol. 18, 145150.

Hurni, H. (1987)," Erosion productivity conservation system in Ethiopia. In I placentae (Ed), soil conservation and productivity", Proc. Of the $4^{\text {th }}$ Int. soil conservation conference. ;654-674.

International Center for Research in the Dry Areas (ICARDA). (2014), "Geo-informatics Unit", http://gu.icarda.org/en.

Kabirigi, M. (2015), "Effects of conservation agriculture on bean and maize yield, soil properties and water productivity in Bugesera District, Rwanda", MSc Thesis. October. P 37.

Karim, M., Fracheboud, Y., Stamp, P. (2000), "Effect of high temperature on seedling growth and photosynthesis of tropical maize genotypes", J. Agron. Crop Sci. 184, 217-223.

Leake, A. R. (2003), "Integrated pest management for conservation agriculture": In Conservation agriculture: environment, farmers experiences, innovations, socio-economy, policy (eds L. Garcia-Torres, J. Benites, A. Martinez-Vilela \& A.Holgado-Cabrera), pp. 271-279. Dordrecht, The Netherlands; Boston, Germany; London, UK: Kluwer Academia Publishers.

Lemma, T., Menfes, T. \& Fantaw, Y. (2015), “Effects of integrating different soil and water conservation measures into hillside area closure on selected soil properties in Hawassa Zuriya District, Ethiopia”, Vol. 6(10), pp. 268-274, October, DOI 10.5897/JSSEM15.0513.

Mehmood, S., Zamir, S., Rasool, T. \& Akbar, W. (2014), "Effect of Tillage and Mulching on Soil Fertility and Grain Yield of Sorghum", Scientia Agriculturae.

Ministry of Agriculture (MOA). (2015), "Ministry of agriculture, Annual report" Retrieved on December.

Musa, H. A. (2014), "Farmer's decision to practice crop rotation in Arsi-negelle, Ethiopia: What are the determinants?", International Journal of Sustainable Agricultural Research, 1(1): 19-27.

Musa, H. A.(2015), "Adoption of multiple agricultural technologies in maize production of the Central Rift Valley of Ethiopia”, Studies in Agricultural Economics 117 (2015) 162-168.

Nath, M.P. (2016), Multiple Cropping for Raising Productivity and Farm Income of Small Farmers", Journal of Nepal Agricultural Research Council. Vol. 2:37-45. DOI: http://dx.doi.org/10.3126/jnarc.v2i0.16120

Rashid, S., Getnet, K. \& Lemma, S. (2010), "Maize Value Chain Potential in Ethiopia: Constraints and opportunities for enhancing the system”, https://www.researchgate.net/publication/282729771.

Sandler, L. \& Kelly, A.N. (2016), "Inter- and Double-crop Yield Response to Alternative Crop Planting Dates", Agricultural Science Volume 4, Issue 2, 01-14.

Semu, A. A. (2018), "The impact of soil and water conservation for improved agricultural production in Ethiopia", J Agri Vol.1 No.1 2018.

Serraj, R.\& Siddique, K.H.M. (2012), “Conservation agriculture in dry areas. Field Crops Res”, 132, 1- 6.

Shiferaw, H. (2005), "Resource degradation and adoption of land conservation technologies in the Ethiopia highland: A case study in Andittid, north shewa agricultural economics", 18:233-47.

Solomon, J. H. (2018), "Risk and opportunities from more productive and resilient cropping system strategies in the Central and Southern Rift Valley of Ethiopia", PhD Thesis. Queensland Alliance for Agriculture and Food Innovation (QAAFI), Queensland.

Thierfelder, C., Cheesman, S. Rusinamhodzi, L. (2012), “A comparative analysis of conservation agriculture systems: Benefits and challenges of rotations and inter-cropping in Zimbabwe", Field Crops Research 137 (2012) 237-250.

Trippathi, R. P, Singh, H.P. (1993), "Soil erosion and conservation in Ethiopia, regional soil conservation unit Swedish, international development authority", Nairobi Kenya.

Tsubo, M., Walker S. \& Ogindo, H.O. (2005), “A simulation model of cereal-legume inter-cropping systems for semi-arid regions", II. Model application. Field Crops Res. 93: 23-33.

Unger, P. W., Langdale, D.W. \& Papendick, R.I. (1988), "Role of crop residues-improving water conservation and use", Cropping strategies for efficient use of water and nitrogen, vol. 51 (ed. W. L. Hargrove), pp. 69 100. Madison, WI: American Society of Agronomy.

Verhulst, N., Govaerts, B., Nelissen, V., Sayre, K.D., Crossa, J., Raes, D., Deckers, J. (2011), “The effect of tillage, crop rotation and residue management on maize and wheat growth and development evaluated with an optical sensor”, Field Crops Res. 120, 58-67.

Yilmaz, F., Atak, M., Erayman, M. (2008), "Identification of Advantages of Maize-Legume Inter-cropping over Solitary Cropping through Competition Indices in the East Mediterranean Region”, Turk J Agric for 32 (2008) 111-119. 